\title{
Clustering-Based Selection for the Exploration of Compiler Optimization Sequences
}

\author{
LUIZ G. A. MARTINS, Federal University of Uberlândia \\ RICARDO NOBRE and JOÃO M. P. CARDOSO, University of Porto \\ ALEXANDRE C. B. DELBEM and EDUARDO MARQUES, University of São Paulo
}

\begin{abstract}
A large number of compiler optimizations are nowadays available to users. These optimizations interact with each other and with the input code in several and complex ways. The sequence of application of optimization passes can have a significant impact on the performance achieved. The effect of the optimizations is both platform and application dependent. The exhaustive exploration of all viable sequences of compiler optimizations for a given code fragment is not feasible. As this exploration is a complex and time-consuming task, several researchers have focused on Design Space Exploration (DSE) strategies both to select optimization sequences to improve the performance of each function of the application and to reduce the exploration time. In this article, we present a DSE scheme based on a clustering approach for grouping functions with similarities and exploration of a reduced search space resulting from the combination of optimizations previously suggested for the functions in each group. The identification of similarities between functions uses a data mining method that is applied to a symbolic code representation. The data mining process combines three algorithms to generate clusters: the Normalized Compression Distance, the Neighbor Joining, and a new ambiguity-based clustering algorithm. Our experiments for evaluating the effectiveness of the proposed approach address the exploration of optimization sequences in the context of the ReflectC compiler, considering 49 compilation passes while targeting a Xilinx MicroBlaze processor, and aiming at performance improvements for 51 functions and four applications. Experimental results reveal that the use of our clustering-based DSE approach achieves a significant reduction in the total exploration time of the search space $(20 \times$ over a Genetic Algorithm approach) at the same time that considerable performance speedups (41\% over the baseline) were obtained using the optimized codes. Additional experiments were performed considering the LLVM compiler, considering 124 compilation passes, and targeting a LEON3 processor. The results show that our approach achieved geometric mean speedups of $1.49 \times, 1.32 \times$, and $1.24 \times$ for the best 10,20 , and 30 functions, respectively, and a global improvement of 7\% over the performance obtained when compiling with -O2.
\end{abstract}

CCS Concepts: • Software and its engineering $\rightarrow$ Compilers; $\bullet$ Mathematics of computing $\rightarrow$ Combinatorial optimization; $\bullet$ Computing methodologies $\rightarrow$ Discrete space search

This work has been partially supported by the FCT (Portuguese Science Foundation) under research grants SFRH/BD/82606/2011 and FEDER/ON2 and FCT project NORTE-07-124-FEDER-000062. LGAM had a scholarship granted by CAPES (process: 0352/13-6). The FEUP authors acknowledge the CoSy license and technical support provided by ACE Associated Compiler Experts bv, The Netherlands.

Extension of Conference Paper. Preliminary versions of this research appeared in the 2014 ACM LCTES under the title "Exploration of Compiler Optimization Sequences Using Clustering-Based Selection," and in the 2014 IEEE CEC under the title "A Clustering-Based Approach for Exploring Sequences of Compiler Optimizations."

Authors' addresses: L. G. A. Martins, Faculty of Computing, Federal University of Uberlândia, Av. João Naves de Ávila, 2121, Uberlândia, MG, 38400-902, Brazil; email: lgamartins@ufu.br; R. Nobre and J. M. P. Cardoso, Faculty of Engineering, University of Porto and INESC-TEC, Rua Dr. Roberto Frias, Porto, 4200465, Portugal; emails: ricardo.nobre@fe.up.pt,jmpc@acm.org; A. C. B. Delbem and E. Marques, Institute of Mathematics and Computer Science, University of São Paulo, Av. Trabalhdor Sãocarlense, 400, Centro, São Carlos, SP, 13560-970, Brazil; emails: \{acbd, emarques\}@icmc.usp.br.

Permission to make digital or hard copies of part or all of this work for personal or classroom use is granted without fee provided that copies are not made or distributed for profit or commercial advantage and that copies show this notice on the first page or initial screen of a display along with the full citation. Copyrights for components of this work owned by others than ACM must be honored. Abstracting with credit is permitted. To copy otherwise, to republish, to post on servers, to redistribute to lists, or to use any component of this work in other works requires prior specific permission and/or a fee. Permissions may be requested from Publications Dept., ACM, Inc., 2 Penn Plaza, Suite 701, New York, NY 10121-0701 USA, fax +1 (212) 869-0481, or permissions@acm.org.

(c) 2016 ACM 1544-3566/2016/03-ART8 $\$ 15.00$

DOI: http://dx.doi.org/10.1145/2883614 
Additional Key Words and Phrases: Clustering, design space exploration, phase-ordering problem

ACM Reference Format:

Luiz G. A. Martins, Ricardo Nobre, João M. P. Cardoso, Alexandre C. B. Delbem, and Eduardo Marques. 2016. Clustering-based selection for the exploration of compiler optimization sequences. ACM Trans. Archit. Code Optim. 13, 1, Article 8 (March 2016), 28 pages.

DOI: http://dx.doi.org/10.1145/2883614

\section{INTRODUCTION}

Due to the large number of optimization passes available in modern compilers and to the specific opportunities for compiler optimizations, an exhaustive exploration of all possible sequences of passes is not feasible. Standard compilers rely on optimization levels that represent fixed optimization sequences. These optimization levels are selected through a simple set of compiler parameters. Several authors have shown that the choice of optimizations, and their order of application, can have a significant impact on performance [Almagor et al. 2004; Hoste and Eeckhout 2008; Jantz and Kulkarni 2013]. Developers typically use their expertise to engage in a labor-intensive source code modification process when the standard optimization does not result in a solution that complies with the design requirements. Design Space Exploration (DSE) is an alternative that can be used to search for specialized sequences of optimization for a given code fragment (or function), considering the target processor and the set of optimizations supported by the compiler. Moreover, stringent time-to-market requirements in embedded systems usually impose restrictions in relation to DSE execution time, and thus, it is desirable to speed up the DSE process as much as possible.

Researchers have investigated several strategies to explore and address the selection and ordering of compilation passes. Iterative compilation techniques usually attempt to find suitable sequences of compiler passes through adaptive search in the optimization space [Cooper et al. 2006; Kulkarni et al. 2010; Chen et al. 2012]. Usually, iterative compilation consists of several compilations of the source code using distinct sequences of the passes and choosing the one that achieves the best performance. Although iterative search can effectively improve the performance of the applications, it still requires a large number of exploration runs. Machine learning and statistical approaches have been used to dramatically reduce the cost of search [Agakov et al. 2006; Fursin et al. 2011; Kulkarni and Cavazos 2012]. They usually involve the use of prediction models trained to select compiler optimization sequences to maximize performance based on code features. The machine-learning approaches significantly reduce the exploration time, whereas methods based on iterative compilation typically obtain codes with better performance [Ashouri et al. 2014].

Recently, we presented the general idea of a DSE approach based on software code clustering to search for optimization sequences aiming at performance improvements of code fragments (e.g., functions) [Martins et al. 2014a, 2014b]. Since the full design space is inviable to explore, we employ a first stage where a clustering method selects a set of relevant compiler optimizations for an input function. Clustering forms a set of functions containing the new input function and the most similar functions out of a reference set previously defined. The identification of similarities between functions uses a data mining method that is applied to a symbolic code representation of the source code, named DNA of the program [Sanches and Cardoso 2010]. The data mining method combines three different algorithms to generate the clusters with benchmarks and the target function: Normalized Compression Distance (NCD) [Cilibrasi and Vitanyi 2005], Neighbor Joining (NJ) [Felsenstein 2003], and a new ambiguity-based clustering algorithm. The reduced optimization set provided by the compiler passes previously suggested for the reference functions in each group is used in the next stage. The DSE approach uses the reduced set identified by clustering in two ways: as the search space or as the initial sequence of the next stage. In both ways, the adoption of a preselected 


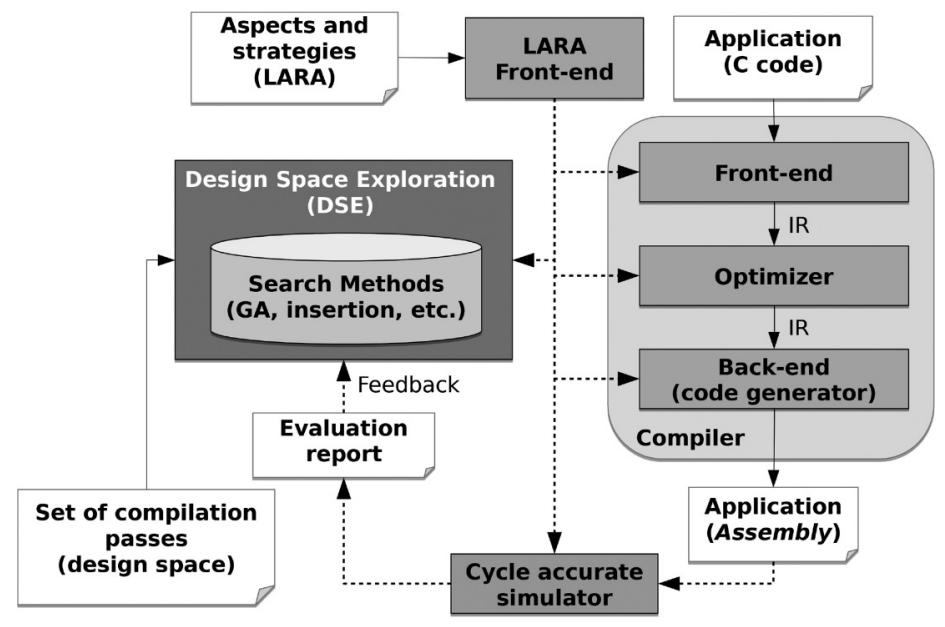

Fig. 1. LARA-based compilation flow.

optimization set allows the use of simple and fast DSE algorithms. Different search algorithms can be applied at the second stage. In this article, we explore a number of alternatives: genetic algorithm, random sampling, cleaning, insertion, and two hybrid approaches that mix the Clean and the Insertion algorithms. Intentionally, our DSE approach selects compilation passes without considering any previous knowledge about their interactions or compilation stage where they are usually employed.

In this article, we present detailed research of the new DSE approach, with a comparative analysis of the evaluated alternative strategies to conclude which one is more adequate for each purpose considering a multiobjective point of view. Besides, we explore several features of the DSE approach, evaluating several design decisions and providing some alternative methods. Finally, a crucial point required for the application of our DSE approach in a practical compiler is the definition of a set of reference functions for which compiler optimization sequences are previously determined. Here we explore different criteria to select a representative set out of a pool of known functions. This research aims to answer the following questions: Is the DNA representation an efficient input? Are the translation rules employed for encoding the DNA representation adequate? Is the DNA based on loop structures enough to guide clustering aiming to achieve an efficient subspace of optimizations? Are the NCD and the bzlib the best combination of distance metric and compressor library to be used to quantify the similarities? What is the more adequate criterion to systematically select representative functions to compose the reference set in order to achieve the best DSE performance?

The rest of this article is organized as follows. Section 2 presents the compilation environment used in this work. Section 3 depicts our clustering-based approach to select compiler passes and introduces the fundamentals of the techniques used for source code representation and for data mining. The algorithms employed in our DSE approach are described in Section 4. Section 5 presents the experiments performed to evaluate our approach and the results achieved. Section 6 reviews related work on the topic of determining sequences of compiler optimizations. Finally, Section 7 presents some conclusions and briefly describes ongoing and future work.

\section{DSE INFRASTRUCTURE}

Our experiments were run on top of an integrated compilation environment fully controlled by LARA [Cardoso, et al. 2012], a domain-specific aspect-oriented programming (AOP) language. Figure 1 shows the LARA-based compilation flow used in this work. 
LARA effectively provides a means for the implementation of DSE strategies able to control and guide compiler tool chains, as well as to evaluate the optimization alternatives based on feedback information reported during exploration. LARA strategies define specific compiler optimizations that best suit the mapping of an application to the target processor. A top-level aspect implements the DSE algorithm relying on the LARA outer-loop mechanism (represented by dashed lines in Figure 1), while other aspects are responsible for guiding the compiler, and for evaluation of the resulting codes. This integrated and unified view allows us to apply different DSE strategies across multiple targets.

In this article, we used two alternative compilation environments: one based on the ReflectC compiler and other based on LLVM.

\subsection{ReflectC-Based Compilation Environment}

This environment was originally developed in the context of the REFLECT FP7 Project [Cardoso et al. 2013] and includes ReflectC, a compiler controlled by LARA aspects/strategies. The ReflectC tool chain built around LARA abstracts the developer from the interaction with all used mapping tools while effectively guiding the DSE flow process in the exploration of alternative optimization sequences targeting the target processor. It is based on CoSy (COmpiler SYstem) [ACE 2012], an industrial compiler that supports a large number of optimizations. ReflectC exposes full control of the application order of optimizations (named as engines) to the LARA weaver and allows optional tuning of those compilation passes. The weaver is responsible for executing the LARA specifications expressing compilation passes to be applied.

Table I shows the 49 ReflectC compilation passes used in our experiments. Some of these optimizations were selected based on their apparent importance in the scope of the REFLECT project. Intentionally, our DSE approach randomly selects optimization passes without considering any knowledge about their applicability with respect to order and compilation stage (analysis, optimizations, and lower passes). More details about the CoSy passes can be found in ACE [2012].

We use Xilinx MicroBlaze as the target processor for ReflectC as it was one of the microprocessors previously used in the REFLECT design flow and for which we have a cycle-accurate simulator. The simulator is configured to simulate an area-optimized three-stage MicroBlaze and to include a barrel shifter, a hardware divisor/multiplier, and the parallel compare instruction. It returns an evaluation report containing the latency in number of clock cycles, which is used as feedback for the DSE loop.

\subsection{LLVM-Based Compilation Environment}

The LLVM compiler infrastructure [Lattner and Adve 2004] is a collection of modular and reusable compiler and tool chain technologies able to support both static and dynamic compilation. We explore compiler sequences with the LLVM optimizer tool (opt) using a total of 124 compiler passes (presented in Table II). The resulting code is fed to the LLVM static compiler $(l l c)$ in order to generate SPARC V8 assembly targeting the LEON3 soft-core processor [Aeroflex 2005]. The LEON3 binaries are then generated with GNU Binutils targeting the SPARC architecture. The code is simulated using the Aeroflex Gaislers TSIM2 cycle-accurate simulator (version 2.0.36) [Aeroflex 2003]. We used the default TSIM2 configuration, which configures a simulated LEON3 core with $4,096 \mathrm{Kbytes}$ of SRAM memory in a single bank, 32Mbytes of SDRAM memory in a single bank, and 2,048Kbytes of ROM memory.

\section{CLUSTERING-BASED PASSES SELECTION}

Sanches and Cardoso [2010] observed that the direct employment of typical clustering methods over source codes is hard due to some intrinsic characteristics mainly related 
Table I. ReflectC Compilation Passes Used in DSE

\begin{tabular}{|c|c|c|c|}
\hline Pass & Description & Pass & Description \\
\hline algebraic & $\begin{array}{l}\text { apply several algebraic } \\
\text { simplifications }\end{array}$ & ckfstrength & $\begin{array}{l}\text { rewrite calls to compiler-known } \\
\text { procedures to cheaper alternatives }\end{array}$ \\
\hline alias & set the Aliased field for objects & blockmerge & merge basic blocks \\
\hline chainflow & jump-to-jump optimizer & constprop & constant propagator \\
\hline cache & $\begin{array}{l}\text { cache memory locations in local } \\
\text { tempos }\end{array}$ & condassigncreate & $\begin{array}{l}\text { replace control dependencies by } \\
\text { conditional assignments }\end{array}$ \\
\hline conevun & $\begin{array}{l}\text { constant evaluation and expression } \\
\text { simplification }\end{array}$ & dismemun & $\begin{array}{l}\text { rewrite the references to arrays as } \\
\text { pointer arithmetic }\end{array}$ \\
\hline copyprop & propagate copies of variables & demote & demote bit width of operations \\
\hline cse & common subexpression elimination & exprprop & expression propagator \\
\hline domorder & $\begin{array}{l}\text { order blocks with the help of the } \\
\text { dominator tree }\end{array}$ & hwloopcreate & $\begin{array}{l}\text { replace the loops for hardware } \\
\text { implementation (loop counters) }\end{array}$ \\
\hline globcse & $\begin{array}{l}\text { global common subexpression } \\
\text { elimination }\end{array}$ & ifconvert & $\begin{array}{l}\text { replace control dependencies by data } \\
\text { dependencies }\end{array}$ \\
\hline funceval & evaluate functional function calls & loopcanon & canonization actions on loops \\
\hline loopbcount & $\begin{array}{l}\text { set UseEstimate of blocks based } \\
\text { on loop nest }\end{array}$ & setrefobj & $\begin{array}{l}\text { set the AbstractValue field of pointer } \\
\text { objects }\end{array}$ \\
\hline loopfuse & fuse two adjacent loops & loophoist & hoist statements from loops \\
\hline loopive & loop induction variable elimination & loopremove & empty loop optimizer \\
\hline looprev & rewrite loop to count down & loopscalar & scalar replacement on loops \\
\hline loopguard & $\begin{array}{l}\text { change while-do loops into } \\
\text { protected do-while }\end{array}$ & loopstrength & $\begin{array}{l}\text { within loops, rewrite pointer expressions } \\
\text { to variables }\end{array}$ \\
\hline mvpostop & $\begin{array}{l}\text { move postoperator assignment } \\
\text { operators }\end{array}$ & lowerboolval & $\begin{array}{l}\text { rewrite Boolean variables to explicit } \\
\text { control flow }\end{array}$ \\
\hline lowerpfc & $\begin{array}{l}\text { translate pure function calls to } \\
\text { normal function calls }\end{array}$ & markconvert & $\begin{array}{l}\text { mark mirConvert when argument is in } \\
\text { range }\end{array}$ \\
\hline misc & miscellaneous optimizations & lrrename & life range renaming of local variables \\
\hline rodata & read-only data & lowerbitfield & rewrite the bit operations as logical \\
\hline noreturn & $\begin{array}{l}\text { remove unreached code after not } \\
\text { returning function call }\end{array}$ & scalarreplace & $\begin{array}{l}\text { replace local structured variables by } \\
\text { scalars }\end{array}$ \\
\hline strength & strength reduction & promote & arithmetic expression size promotion \\
\hline tailrec & eliminate tail recursion & setpurity & compute side effect flags for procedure \\
\hline tailmerge & merge common tails & loopinvariant & perform invariant code motion on loops \\
\hline vprop & $\begin{array}{l}\text { evaluation and propagation of } \\
\text { expressions }\end{array}$ & vstrength & $\begin{array}{l}\text { strength reduction based on value range } \\
\text { analysis }\end{array}$ \\
\hline vshrink & reduce size of local variables & & \\
\hline
\end{tabular}

to their dependence of the code features. These difficulties are overcome in our DSE approach by using a symbolic representation of the target code and employing a variation of the data mining method proposed in Sanches et al. [2011]. This approach allows one to find patterns in the translated codes independent of the code size or programming language. The data mining method combines three different algorithms to generate the clusters with reference benchmarks and a given input function (target): NCD [Cilibrasi and Vitanyi 2005], NJ [Felsenstein 2003], and a new ambiguity-based clustering algorithm.

Basically, the clustering stage is composed of five steps: (1) the source code of the target function is translated to a symbolic representation, referred to as DNA of a program [Sanches and Cardoso 2010] - this new translated code is included in the set formed by the preprocessed DNA codes of several reference functions; (2) the distance matrix is calculated over this set of DNA codes by using the NCD [Cilibrasi and Vitanyi 2005 ] for each pair of codes; (3) the tree topology is constructed from the distance matrix by using a phylogenetic reconstruction algorithm, named NJ [Felsenstein 2003]; (4) the 
Table II. LLVM Compiler Passes Used in DSE

\begin{tabular}{|c|c|c|c|c|}
\hline -aa-eval & -adce & -add-discriminators & -alloca-hoisting & -always-inline \\
\hline -argpromotion & -atomic-ll-sc & -barrier & -basicaa & -basiccg \\
\hline -bb-vectorize & -block-freq & -bounds-checking & -branch-prob & -break-crit-edges \\
\hline -codegenprepare & -consthoist & -correlated-propagation & -constprop & -cost-model \\
\hline -constmerge & -count-aa & -da & -dce & -deadargelim \\
\hline -debug-aa & -debug-ir & -delinearize & -dfsan & -die \\
\hline -domfrontier & -domtree & -dse & -early-cse & -extract-blocks \\
\hline -functionattrs & -generic-to-nvvm & -globaldce & -globalsmodref-aa & -globalopt \\
\hline -gvn & -indvars & -inline & -inline-cost & -instcombine \\
\hline -instcount & -instnamer & -instsimplify & -internalize & -intervals \\
\hline -ipconstprop & -ipsccp & -iv-users & -jump-threading & -jump-instr-table-info \\
\hline -lazy-value-info & -lcssa & -libcall-aa & -licm & -lint \\
\hline -load-combine & -loop-deletion & -loop-extract & -loop-idiom & -loop-reduce \\
\hline -loop-reroll & -loop-rotate & -loop-extract-single & -loop-instsimplify & -loop-simplify \\
\hline -loop-unroll & -loop-unswitch & -loop-vectorize & -loops & -lower-expect \\
\hline -loweratomic & -lowerinvoke & -lowerswitch & -mem2reg & -memcpyopt \\
\hline -memdep & -mergefunc & -mergereturn & -metarenamer & -mldst-motion \\
\hline -module-debuginfo & -notti & -nvptx-favor-non-generic & -nvvm-reflect & -nvptx-assign-valid-global-names \\
\hline -objc-arc & -objc-arc-aa & -objc-arc-apelim & -objc-arc-expand & -objc-arc-contract \\
\hline -partial-inliner & -postdomtree & -partially-inline-libcalls & -prune-eh & -reassociate \\
\hline -reg2mem & -regions & -sample-profile & -scalar-evolution & -scalarizer \\
\hline -scalarrepl & -scalarrepl-ssa & -simplifycfg & -scep & -scev-aa \\
\hline$-\operatorname{sink}$ & -slp-vectorizer & -strip-dead-debug-info & -sroa & -separate-const-offset-from-gep \\
\hline -strip & -strip-dead-prototypes & -strip-nondebug & -structurizecfg & -strip-debug-declare \\
\hline -tailcallelim & -targetlibinfo & -tbaa & -verify & \\
\hline
\end{tabular}

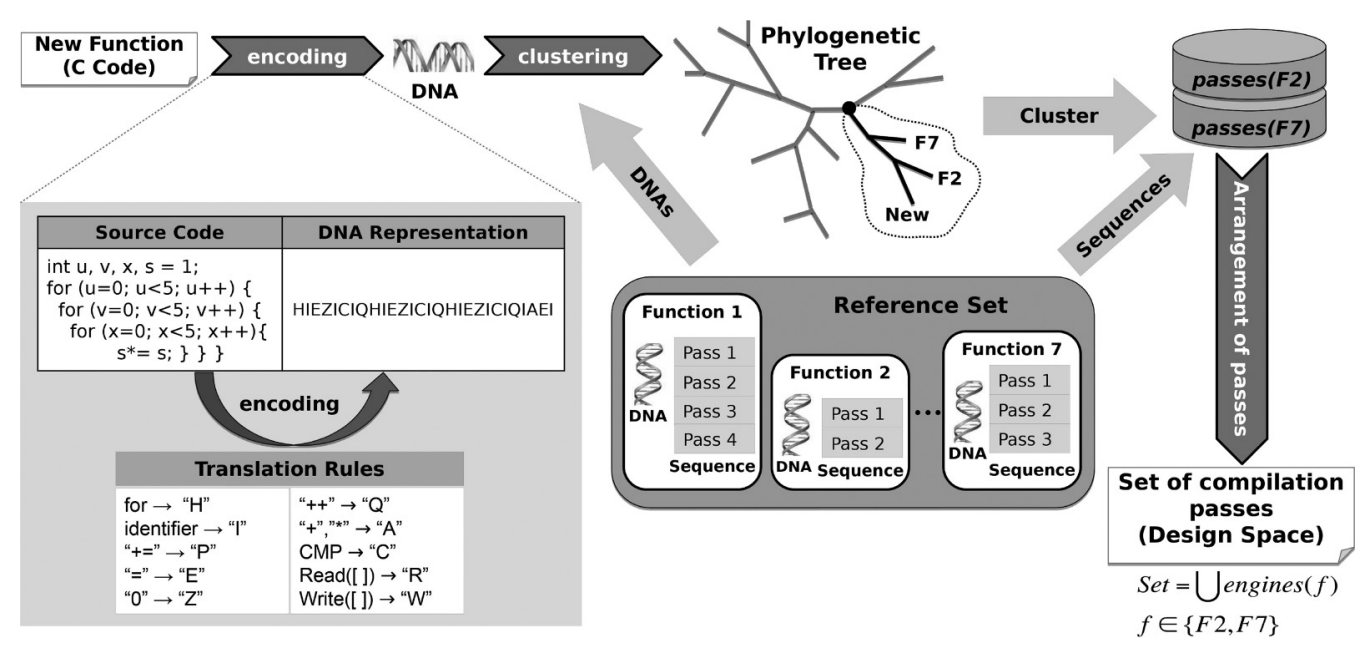

Fig. 2. Selection of compiler engines based on clustering.

ambiguity-based clustering algorithm detects highly correlated groups of functions, extracting potential clusters hidden in the tree topology; and (5) the distinct passes used in optimization sequences for the clustered reference functions are included in the design space employed in the next stage of DSE.

The exploration can thus be performed using any search method implemented in the DSE environment. Figure 2 illustrates the whole clustering process enabling the selection of potential optimizations. This process is responsible for outputting the set of compilation passes adopted in Figure 1 using function granularity as an example. The steps and techniques employed in this clustering process are described as follows. 


\subsection{DNA Encoding}

The identification of patterns over source code can be a very complex task, as it may need to deal with multiple files, lines of code, and code structures of different shapes and dimensions. Most pattern-mining research [Wheeler and Kececioglu 2007; Roy et al. 2009] relies on high similarity levels that hamper the identification of code patterns with subtle differences, but that possibly share the same optimization strategy.

We employ a symbolic encoding due to its capability to highlight main program structures, allowing us to identify approximate patterns from the source code. The DNA is a symbolic representation proposed in Sanches and Cardoso [2010], where program elements (e.g., operators and loops) are encoded in a sequence of symbols. The encoding process is based on translation rules and generates lossy intermediate representations. According to the translation rules used, this representation may reflect with higher or lower degree the sequence of tokens that identifies each program/function.

Initially, we use the same translation rules employed in Sanches et al. [2011], which are highlighted in the DNA encoding example presented in Figure 2. The authors claim that this rule set emphasizes the similarities in the loop body operations. Other translation rules are explored in the experiments reported in Section 5.

\subsection{Normalized Compression Distance}

The NCD is a compression-based metric proposed in Cilibrasi and Vitanyi [2005], whose properties are based on Kolmogorov complexity theory [Li and Vitanyi 1997]. The basic idea is that two objects are considered close if one can be significantly compressed using the information in the other. Therefore, the distance $N C D(x ; y)$ for each pair of functions ( $x$ and $y$ ) is the improvement due to compressing $y$ using $x$ as reference instead of compressing $y$ from scratch, expressed as the ratio between the bitwise length of the two compressed versions. It can be calculated as follows [Cilibrasi and Vitanyi 2005]:

$$
N C D(x ; y)=\frac{C(x y)-\min (C(x) ; C(y))}{\max (C(x) ; C(y))},
$$

where $C(\star)$ is the length of the compressed version of the file $\star$, and $x y$ is the file resulting from the concatenation of $x$ and $y$.

The algorithm computes the NCD metric from the DNA code, generating the matrix corresponding to the references and target functions. Many compressors can be used in NCD computation. We initially adopted the bzlib compressor for calculating the input a space between distance and matrices [Martins et al. 2014a]. Other compressors were evaluated in experiments reported in Section 5.

The NCD metric does not require any knowledge about the features of the data analyzed to find relationships. Such feature independence enables the NCD-based algorithm to deal with several types of program data at code level, including (but not limited to) the source codes and intermediate representations (as DNA). However, other distance metrics can be applied as shown in Section 5 .

\subsection{Neighbor Joining Algorithm}

The NJ [Felsenstein 2003] is a simple and computationally efficient algorithm for constructing phylogenetic trees. The algorithm output is an unrooted tree structure that describes the relationships among objects. The tree topology is defined by consecutively joining pairs of neighbors. This tree is iteratively constructed starting from a star-like tree, where all objects (leaf nodes) are connected through one interior node. At each iteration, a new pair of neighbors is connected by an intermediate node and the distance matrix is recalculated considering this new node. The algorithm provides a tree topology that enables determining recursively clusters composed by other clusters. Finally, 
as NJ does not depend on any type of a priori knowledge about the problem domain, it is an interesting algorithm for identifying hierarchical similarities among software codes [Sanches et al. 2011].

\subsection{Ambiguity-Based Clustering}

In order to extract potential clusters hidden in the tree topology, it is necessary to identify subtrees with a high degree of independence in the generated phylogenetic tree. A hierarchical clustering algorithm is employed here for this identification. The Fast Newman algorithm (FN) [Newman 2010] was used in the approach presented in Sanches et al. [2011]. FN is an algorithm from complex networks analysis focused on discovering clusters in large-scale networks. In our DSE approach, we adopt a new clustering method inspired by the measure of ambiguity of tree structures [Martins, et al. 2014a]. In exploratory experiments, it was possible to verify that our ambiguitybased approach works better with small networks than FN.

Tree ambiguity can be defined from the ambiguity between two leaf nodes. Leaves $i$ and $j$ of a tree $T$ are ambiguous if there are two or more internal nodes (ancestors) between them in $T$, that is, internal nodes in the unique path from $i$ to $j$. In other words, both nodes $(i$ and $j$ ) are not tightly similar, since they require more than one intermediate node to counterbalance their differences in relation to other leaf nodes (that compose a third momentum affecting the equilibrium between $i$ and $j$ ). The ambiguity of $T$ (or tree ambiguity) is the accumulated ambiguity involving all the pairs of leaves of $T$. This definition can result in imprecise evaluation of the reliability of a tree, especially when evaluating small trees such as those used in our experiments. We bypass this problem using the concept of strong ambiguity. Basically, two leaf nodes are strongly ambiguous if there are three or more internal nodes (ancestors) between them. Our clustering approach employs measures of strong ambiguity to separate the functions into groups. The number of internal nodes considered can also be modified for tuning the strength of a relationship to establish a cluster. Here, the target function is joined to all functions reachable by up to three intermediate nodes.

\section{DSE STRATEGIES}

We use a number of DSE strategies to perform the search for specialized optimization sequences for a given function using our LARA-based environment. These strategies differ with respect to the technique and the design space employed in the search. Some of them are applied to the entire search space composed by all the possible optimization passes considered in our experiments; other ones are applied to a reduced search space generated by combinations between optimizations returned by the selection method; and, finally, two of them use the reduced space in a first stage and the full space in a second stage. A brief description of each DSE strategy is given as follows.

\subsection{Genetic Algorithm}

The Genetic Algorithm (GA) [Goldberg 1989] is a well-known metaheuristic usually applied to a variety of search problems. It is an evolutionary search method that consists of generating an initial population of random solutions with a subsequent iterative evolution of their individuals, determined by their evaluation and ranking. Each evolutionary step is called generation and is formed by simple procedures: (1) the selection of the parent solutions (sampled pairs of solutions), (2) the application of the crossover and mutation operators, (3) the evaluation of new solutions generated after the operators, and (4) the reinsertion procedure that decides the survivors for the next generation. This iterative process is performed until achieving a stop criterion.

Considering the compiler optimization sequence search, each individual or solution is a sequence in the search space represented as an array with variable size (sequence 
length). The evaluation is performed by the fitness function defined by the number of clock cycles needed to execute the code generated by applying the optimization sequence.

The GA-based approach is performed in both full and reduced exploration spaces. In our experiments, GA runs over a population of 300 individuals when the full space is used and over a population of 30 individuals if the reduced space is used. A uniform distribution was used to generate the initial population of sequences in order to guarantee similar quantities for all possible lengths (from one to 16 compilation passes). In each generation, a uniform crossover operator was defined with $60 \%$ probability for applying crossover to a pair of individuals. A simple tournament selection (Tour $=3$ ) was used for parent solution selection. A mutation rate of $40 \%$ was applied over the population of new solutions generated by crossover. Four types of mutation operators were developed: including a new pass to a point randomly chosen of the compilation sequence; removing the optimization placed in any point of the sequence; exchanging a pass of the sequence by another chosen randomly from the space; and changing the order of two passes of the sequence. The elitism reinsertion strategy keeps the best optimization sequences from parent and children sets in the current population. The stop criteria used are a maximum number of generations (equal to 100) or if 15 subsequent generations do not result in a latency improvement.

\subsection{Random Sampling}

A simple random-sampling-based algorithm is used for the exploration of both full and reduced optimization spaces. The generation of each sample is performed by the same procedure used for the generation of the initial population on the GA approach. In our experiments, the number of samples generated is exactly 10 times the size of the population used in the GA approach: 3,000 sequences when the full space is used and 300 solutions for the reduced space. These sizes are compatible with the number of different solutions evaluated by GA runs on average, since the majority of them stop before 20 generations and $60 \%$ of the population size is created at each generation. Therefore, the random sampling method can give us a reference if no strategy is used in the search and only isolated points are evaluated as potential optimization sequences.

\subsection{Insertion Algorithm}

This approach was proposed in Huang et al. [2013] to deal specifically with the search of optimization sequences. It is an iterative greedy algorithm that incrementally includes new optimizations to a sequence. The algorithm starts by acquiring the performance of the code resulting from compilation of the input function without optimizations. Then, the algorithm traverses the search space of optimizations sequentially, in a predefined order, building a solution by inserting one optimization at a time and verifying the resultant performance of the optimized code. If an improvement occurs, the optimization is included in the current solution. Otherwise, it is discarded and the next optimization is tested. Insertion is applied in both full and reduced design spaces with a slight difference in the predefined order used to choose the optimization operation to be tested in each attempt to include in the current sequence: using the full space, the sequence order is arbitrarily defined, while if the reduced space is used, the order is defined with respect to the proximity among the functions in the phylogenetic tree. In both spaces (full and reduced), when testing the insertion of a new optimization, the algorithm evaluates it in all possible positions of the current sequence, aiming at achieving the best sequence.

Since the optimizations are processed in the same order they appear in the set of compilation passes to explore (search space), the results can be influenced by the arrangement used. For minimizing this problem, it can be useful to traverse that search 
space a number of times. The number of iterations is an input of the algorithm. Here, we adopted three iterations for both approaches (full and reduced space), since higher values did not provide better results in our experiments. The algorithm also stops when there is no improvement between subsequent iterations.

\subsection{Clean Algorithm}

The cleaning algorithm is also an iterative greedy approach, which incrementally removes optimizations from the current sequence. It was first presented in Martins et al. [2014b]. Unlike the Insertion algorithm, Clean starts from a predefined sequence. In each iteration, an optimization is removed from the current sequence in order to verify its effect on the performance of the optimized code. The removal of optimizations is done sequentially, that is, from the first until the last one ( $N$ th pass). It was applied only to the reduced design space, where the sequence (the optimizations and their order) is defined based on the proximity among the functions in the phylogenetic tree. That is, we adopt the same optimization sequence of the closest reference function, including the distinct (new) passes found in the optimization sequences of the other functions in the cluster.

\subsection{Hybrid Approaches}

Two hybrid approaches are used: the Clean-Insertion (CI) and the Insertion-Clean (IC) algorithms. Both of them use more than one stage, and some operations are applied over the reduced space and others over the full space.

The first stage of the Clean-Insertion algorithm starts with the sequence of optimizations generated through the clustering-based selection method. However, it starts applying the Clean algorithm over this sequence removing all superfluous optimizations and reducing the solution to an intermediate sequence. This result is used as the initial sequence in the second stage, where the Insertion algorithm is applied over the full design space for just one iteration step. Since the insertion process starts from a promising reduced solution, the new intermediate sequence can be achieved through inclusion of fewer optimizations from the full optimizations set. Finally, the inclusion of a new optimization can turn others unnecessary in the current sequence. Therefore, the Clean algorithm is performed in the third stage starting from the new intermediate sequence to find the final solution.

The first stage of the Insertion-Clean algorithm also starts with the sequence of optimizations generated through the clustering-based selection method. The Insertion algorithm is performed over the full space for just one iteration step by including new optimizations for generating an intermediate sequence. In practice, the Insertion algorithm allows the exploration of regions neighboring the initial sequence in the full design space. The inclusion of a new optimization can turn another one unnecessary in the current sequence. This intermediate result is used as the initial sequence in the second stage, where the Clean algorithm is applied in order to remove all superfluous optimizations, reducing the solution to an optimal sequence. The basic idea of the IC algorithm is that promising passes can be combined with new optimizations improving the execution time of the program. On the other hand, this approach often deals with larger optimization sequences, where there are more positions for testing a given optimization pass. Therefore, its execution time is excessively larger than the CI approach while the final latencies are very similar.

Examples of the application of the two hybrid approaches are shown in Figure 3. The remaining passes from the initial sequence are highlighted in the output sequences of the algorithms. In those examples, we can see that the final result is exactly the same sequence. 


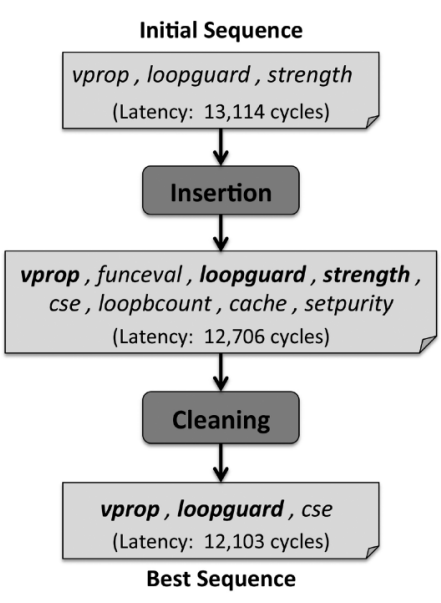

(A) Insertion-Clean (IC) Algorithm

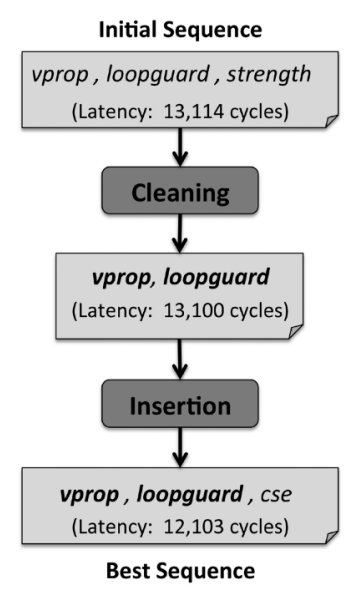

(B) Clean-Insertion (Cl) Algorithm

Fig. 3. Hybrid DSE strategies.

\section{EXPERIMENTAL RESULTS}

Initially, the DSE strategies evaluated in this article were implemented to search for suitable sequences of optimizations targeting a Xilinx MicroBlaze processor (a RISC architecture) for 46 functions from DSP [Texas Instruments 2003a] and image [Texas Instruments 2003b] benchmark repositories from Texas Instruments (TI). A new multiobjective analysis was incorporated for selecting the best DSE strategies. The clustering process was automated and generated a large number of clusters to be analyzed. In addition, the optimization sequences used for each reference function clustered with a target function were tested in order to evaluate the need for joining their optimization passes. Sections 5.3 to 5.5 refer to additional experiments for analyzing in detail the various design decisions used in the clustering, investigating their suitability for the selection of optimization passes from the reference set. Initially, the reference set is formed based on functions described in Sanches et al. [2011]. As a representative selection of this set is very important for the success of the approach, some selection strategies of reference functions are explored in Section 5.6. Section 5.8 refers to additional experiments performed using functions from other computing domains and applications. In Section 5.9, we use our compilation environment targeting the LLVM compiler and a LEON3 soft-core processor, and our approach performance is compared with the -O2 option and with the best sequences present in Purini and Jain [2013].

\subsection{Analysis of DSE Strategies}

We applied a variety of algorithms to explore the optimization space, providing distinct accuracies and execution times, aiming at validating the utility of the clustering-based optimization selection approach in the DSE process. These strategies basically differ with respect to the technique employed in the search of the best optimization sequence for a given function. DSE strategies using random sampling (RS), Insertion (Ins), and genetic algorithm (GA) were executed on the full and reduced optimization sets, whereas the approach based on a cleaning algorithm (Clean) was only executed on a reduced search space. Furthermore, we include two mixed approaches joining the Clean and full Insertion algorithms (CI and IC). The purpose of these hybrid approaches is to overcome the limitations of the original algorithms, providing a more effective exploration of the search space. 


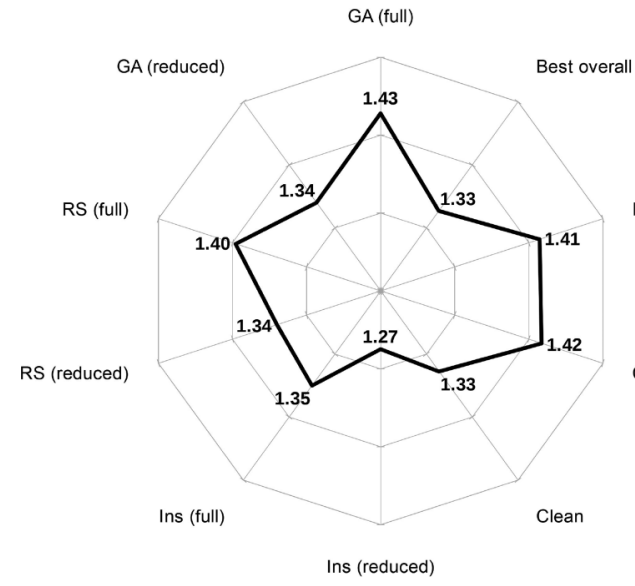

(a) Best speedups

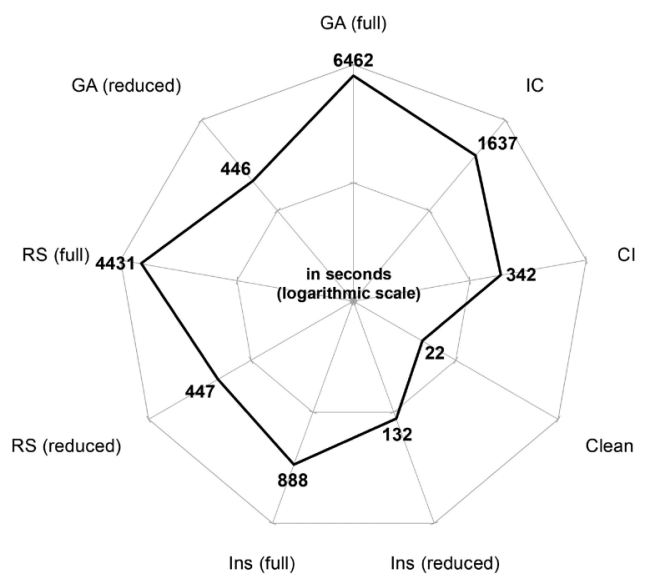

(b) Execution time of the DSE

Fig. 4. Geometric means of the performance metrics according to the DSE strategies.

The experiments without optimization preselection, named Full DSE, were performed using a total of 49 CoSy passes in the search space (see Table I). Given that a maximum sequence length of 16 compiler passes (empirically chosen from previous experiments) was adopted, the cardinality $\eta$ of the search space is given by $\sum_{i=0}^{16} 49^{i}$. Therefore, the number of potential sequences in the search space is $49^{17}-1$, preventing an exhaustive exploration.

The executions with our clustering-based selection approach are identified as Reduced DSE. It was performed using a subset of optimizations (13 passes on average) chosen from the similarity between the target function and reference functions. During the clustering, we considered 11 reference functions [Texas Instruments 2003a; Guthaus, et al. 2001]: adpcm (coder and decoder), autocorrelation, bubble sort, dotprod, fdct, fibonacci, max, min, pop count, and sobel. This reference group is a set of the functions previously used in other contexts [Sanches et al. 2011] and was intentionally kept as it represents functions with different characteristics.

The results obtained from the reduced subset were compared with those achieved with the DSE approaches over the full optimization space. Considering that a GA generally results in values close to optimal [Kulkarni et al. 2007], the speedups achieved by the GA-based DSE with full optimization space are used as the goal for the other strategies. Furthermore, as in the approach used in Kulkarni and Cavazos [2012], we also searched the best single optimization sequence for our 11 reference functions. During this process, each potential optimization sequence was evaluated over all functions, and a geometric mean was calculated from their speedups considering the performance of the original code (without optimizations). The "best overall sequence" was applied over the functions analyzed in order to validate the representativity level of the reference set. The geometric means of the performance metrics achieved for the DSE strategies are shown in Figure 4.

Figure 4(a) presents the geometric means of the best speedups achieved for each DSE strategy and "best overall sequence." These geometric means were calculated with respect to execution clock cycles achieved by the functions without optimizations, that is, from the best speedups over baseline code. In general, the exploration with full space achieved better speedups than when applied in the pruned optimizations set. As expected, the use of the DSE strategy based on GA for the two optimization spaces (full and reduced) achieved the best speedups, and the GA using the full space 


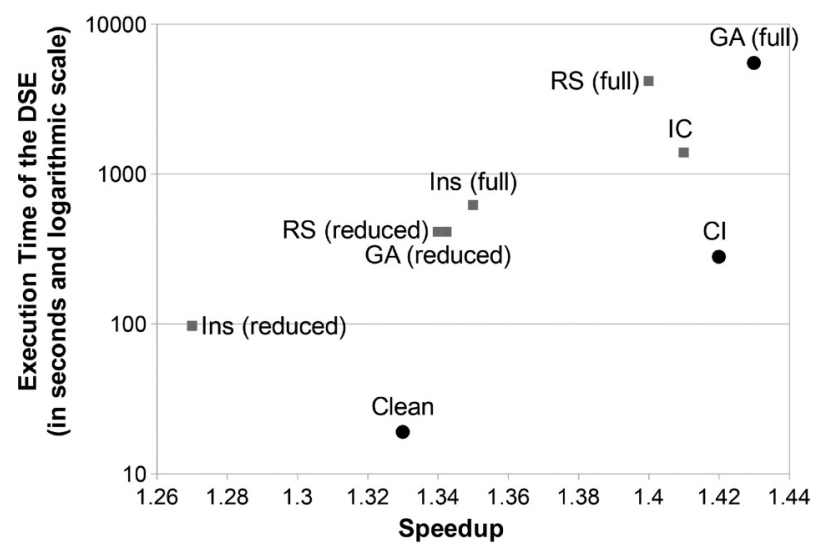

Fig. 5. Performance of the DSE strategies through a multiobjective perspective.

was the best available strategy, resulting in higher values of speedup for all target functions. On the other hand, the Insertion algorithm using a pruned space obtained the worst results, whereas the DSE based on insertion with the full space achieved a close mean speedup to other search approaches with the reduced set. The DSE strategies based on random sampling achieved speedups close to the ones obtained by GA-based approaches. However, RS approaches need to evaluate a solutions set $10 \times$ greater than the one explored by the GA in both spaces. The Clean algorithm is the simplest and fastest approach used in this article. However, its achieved speedups are very close to the GA and random sampling approaches using pruned space and the full Insertion algorithm, and only different in about 0.1 to the full GA-based method. The hybrid approaches achieved speedups very close to those achieved by the GA with full space. The adoption of the "best overall sequence" also achieved good speedups, although its mean speedup is equal to Clean and slightly worse than the other ones (except Insertion reduced). Basically, the approaches can be clustered into three groups according to their speedups. The first group is formed by GA (43\%), Clean-Insertion (42\%), Insertion-Clean (41\%), and random sampling using full space (40\%). The intermediate group comprises the Insertion algorithm with full space (35\%), GA and random sampling using reduced space (both approaches with 34\%), and Clean algorithm and best overall sequence (both with 33\%). The last group is formed by the Insertion algorithm with a pruned space $(27 \%)$. Note that the difference between the best and the worst geometric mean speedups is around 0.16 .

Figure 4(b) presents the average execution time for each DSE strategy. As can be observed, significant improvements were achieved with respect to the execution time when comparing DSE in full and reduced spaces. The GA-based exploration over full space took on average 5,509 seconds, while random sampling, GA, Insertion, and Clean approaches using pruned search spaces took about 422, 412, 97, and 19 seconds, that is, improvements around $13 \times, 13 \times, 57 \times$, and $290 \times$, respectively. Considering the hybrid approaches, the DSE execution time improvements over the GA-based approach were around $20 \times$ and $4 \times$ for the Clean-Insertion and the Insertion-Clean algorithms, respectively.

Figure 5 shows the different DSE strategies compounding the two tradeoff objectives in a single chart, enabling us to identify the dominated and the nondominated strategies in a Pareto-based analysis. Given two strategies ( $a$ and $b$ ), we say that $a$ dominates $b$ if (1) $a$ is not worse than $b$ in all evaluated objectives, and (2) $a$ is better than $b$ in at least one objective. The set of nondominated strategies consists of those ones without 


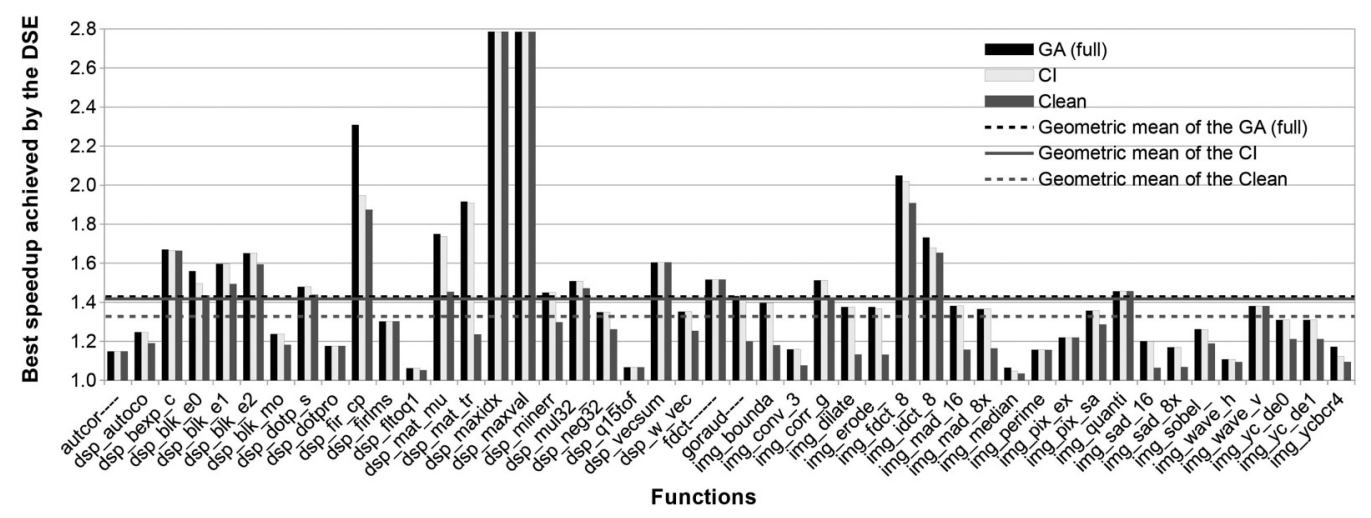

Fig. 6. Speedups obtained for each benchmark using nondominated DSE strategies.

no other strategy dominating them. Since the average execution time presented a severe variation between the minimum (19 seconds) and the maximum (5,509 seconds) values, it is presented using a logarithmic scale. It is possible to observe in this chart that the nondominated front is formed by three strategies, which were plotted using black circles: GA full, CI, and Clean. All the other strategies (gray squares) are dominated by at least one element of the nondominated front. For example, although IC is not dominated by GA full and Clean strategies, it is dominated by CI. Therefore, it can be discarded from the nondominated set. Between the three nondominated solutions, GA full is the one that returns the best geometric mean speedup, but it demands a high processing time. On the other side, Clean is the fastest strategy but returns one of the worst speedups considering all the evaluated methods. CI is the most balanced nondominated solution, since it returns a mean speedup very close to the best one and demands a processing time much lower than the full-space approaches.

Figure 6 outlines the best speedups achieved with the nondominated DSE strategies. To facilitate analysis, we include lines in the chart representing the geometric mean of each DSE strategy. Analyzing function sequences, we observed that (1) the current reference set does not include compilation passes specifically used for some target functions, such as mupostop and globcse used for img_ycbcr4; (2) other ones are used in a few reference sequences (one or two), making difficult its presence in the reduced subsets (e.g., loopfuse and setpurity); and (3) simple algorithms have difficulties in generating sequences with repetitions of passes, which are necessary to optimize the functions $d s p \_b e x p \_c$ and $d s p \_f i r \_c p$.

Figure 7 presents the size of the initial search space explored in reduced approaches, as well as the number of optimizations contained in near-optimal sequences achieved by DSE approaches based on GA (using full space), Clean-Insertion (CI), and Clean algorithms for each function evaluated. In addition, the last bars in Figure 7 show the respective average values. Generally, the final sequences are smaller than the size of initial space, except for $d s p \_b e x p \_c, i m g \_d i l a t e, i m g \_f d c t \_8$, and img_idct_8. Also, the final compilation sequences achieved by GA and CI algorithms are very similar for most functions, justifying their similar performance. Therefore, the reduction in terms of speedup may be acceptable, especially when considering the gains in DSE execution time, as shown in Figure 8.

There are large differences of DSE execution times between the GA-based approach and other DSE strategies. Although the GA-based approach achieves always equal or better optimization sequences, it also has a longer execution time. On the other hand, the Clean algorithm is the fastest approach. The DSE based on CI presents the 


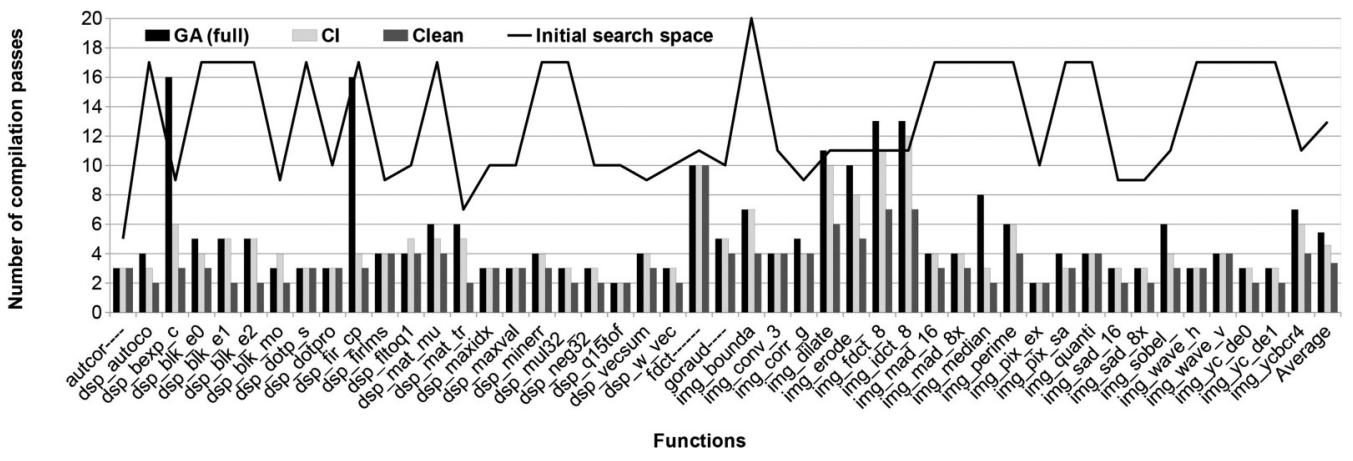

Fig. 7. Sequence lengths in the initial search space and in the final optimization sequences in relation to the search algorithm used and the function evaluated.

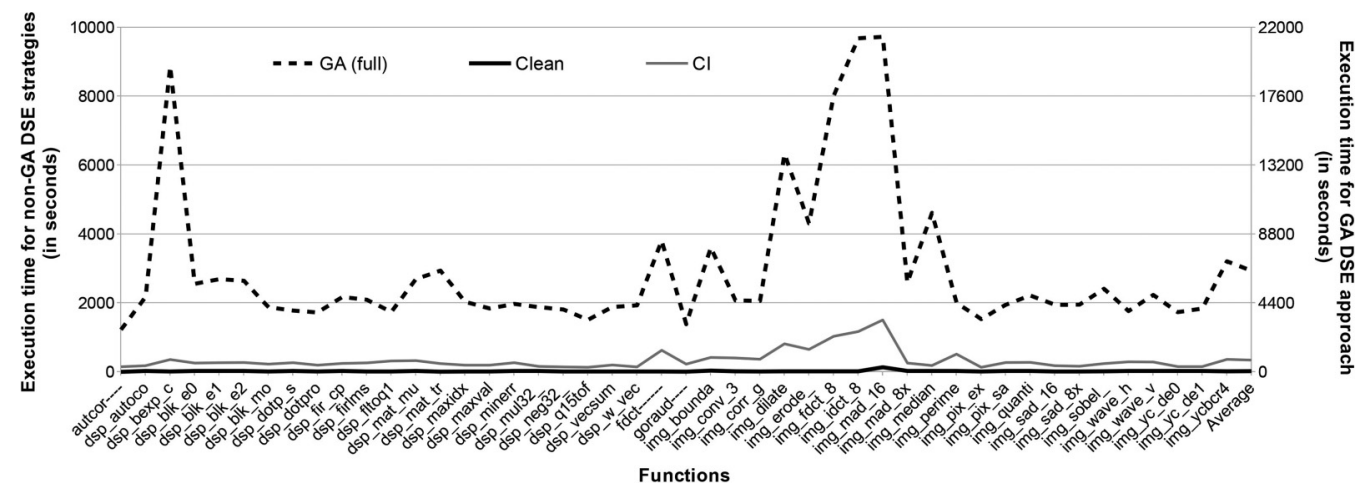

Fig. 8. Execution time of the DSE strategies obtained for each target function evaluated.

intermediate execution time, though closer to Clean. This behavior is observed in the individual analysis of the functions, and in the average execution time of the DSE (last value in Figure 8).

\subsection{Clustering Analysis}

In order to evaluate the effectiveness of the clustering, we perform the reduced DSE from each cluster used during the experiments (12 clusters). The grouped functions and the number of distinct passes contained in each cluster are presented in Table III. Cluster 9 consists of all 20 compiler passes found in sequences associated with the reference functions. So we call it a reference cluster. Clusters 2, 3, 11, and 12 have the same compiler passes, just changing their order in the generated subset. The passes used by the functions in each cluster were adopted as search space by the DSE strategy during the exploration.

Figure 9 presents the geometric means of the speedups achieved with Clean and CI for all 46 TIs' functions considering the compiler passes in each cluster. As can be observed in Figure 9(a), our clustering-based approach achieves the same speedup of the reference cluster $(1.33 \times)$ in Clean. The employment of the reference cluster as search space is able to find the best achievable speedups in the DSE with reduced space. In CI (Figure 9(b)), our selection method obtained the best result between the clusters $(1.42 \times)$, outperforming the value achieved by Cluster 9 (reference).

Considering each function individually, our clustering-based approach did not achieve the best speedup for only four (out of 46) functions. Figure 10 presents the best 
Table III. Clusters Used in the Experiments

\begin{tabular}{|c|c|c|}
\hline Clusters & Reference Functions & \# Passes \\
\hline 1 & autcor, bubble_sor, pop_cnt & 5 \\
\hline 2 & autcor, pop_cnt, sobel, fdct, adpcm_code, adpcm_deco,bubble_sor & 17 \\
\hline 3 & bubble_sor, sobel, fdct,adpcm_code, adpcm_deco & 17 \\
\hline 4 & dotprod, dsp_minval, max, fibonacci & 10 \\
\hline 5 & dsp_minval,bubble_sor & 7 \\
\hline 6 & dsp_minval, dotprod,bubble_sor & 9 \\
\hline 7 & dsp_minval, fibonacci, bubble_sor & 9 \\
\hline 8 & dsp_minval, max, bubble_sor & 10 \\
\hline 9 & dsp_minval, sobel, fdct,adpcm_code, adpcm_deco,bubble_sor & 20 \\
\hline 10 & fdct, sobel & 11 \\
\hline 11 & sobel, fdct, adpcm_code, adpcm_deco & 17 \\
\hline 12 & sobel, fdct, bubble_sor, adpcm_code, adpcm_deco & 17 \\
\hline
\end{tabular}

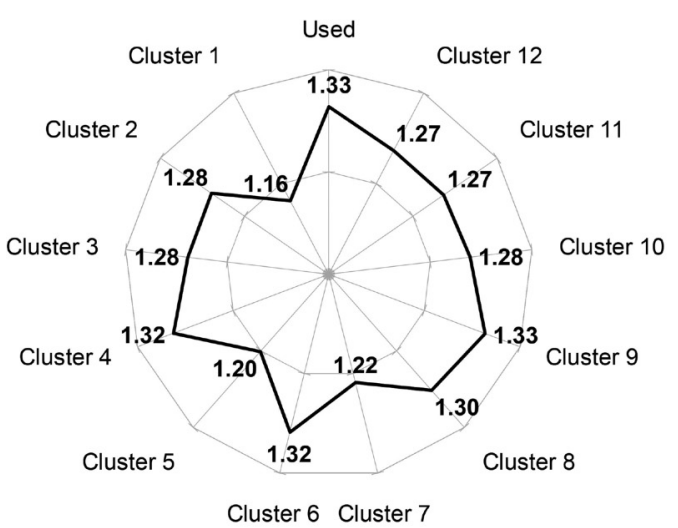

(a) Clean

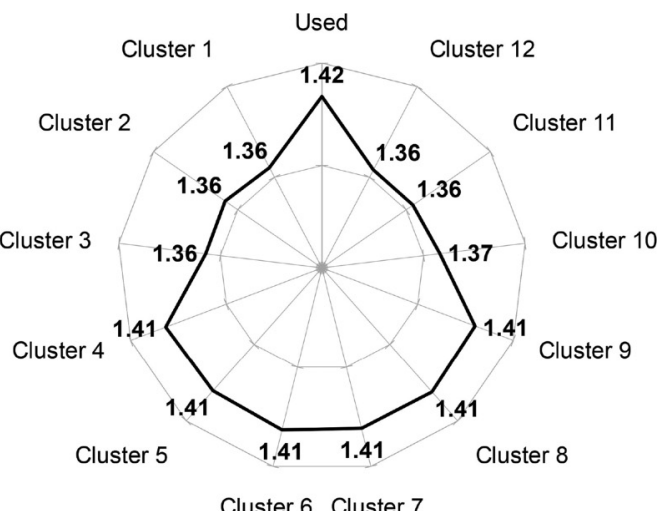

Cluster 6 Cluster 7

(b) Clean-Insertion

Fig. 9. Geometric mean of the speedups according to the cluster used.

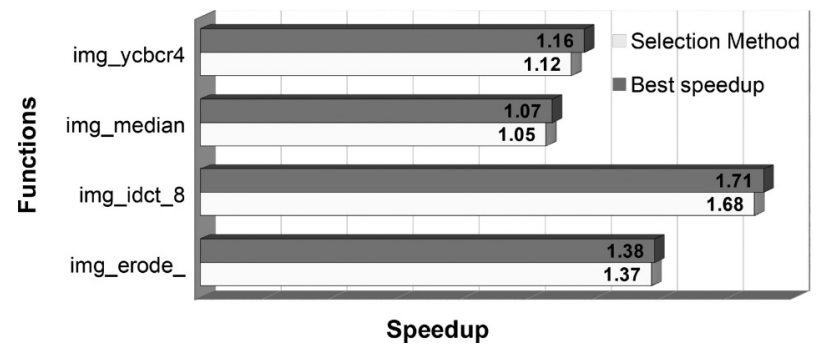

Fig. 10. DSE performance for the functions with which our clustering-based approach did not achieve the best speedup.

speedup achieved by the DSE schemes for these functions, considering the reduced search space provided by each cluster. As can be noted, our approach achieves values very close to the best ones (maximum difference is 0.04 ).

In addition to cluster exploration, we also research the individual optimization sequences used by each reference function in the cluster. This experiment aims to evaluate the influence of the sequences' aggregation in the DSE process. The test was performed using two different approaches, which differ by how the sequence is chosen within the cluster: (1) the sequence of the closest reference function, according to the phylogenetic tree, is applied directly to the new function, and (2) all sequences of the cluster are 


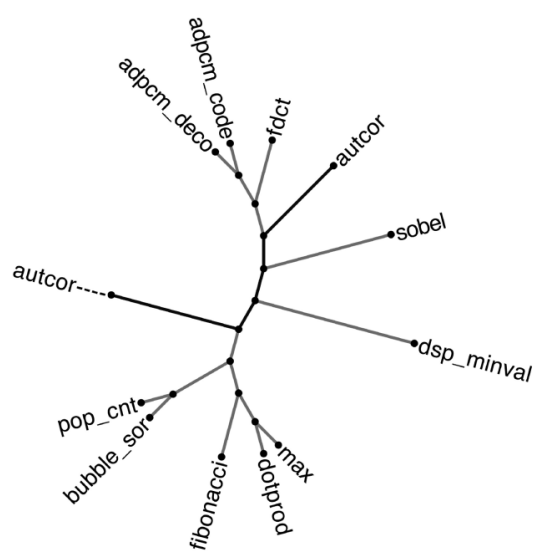

(a) Source code

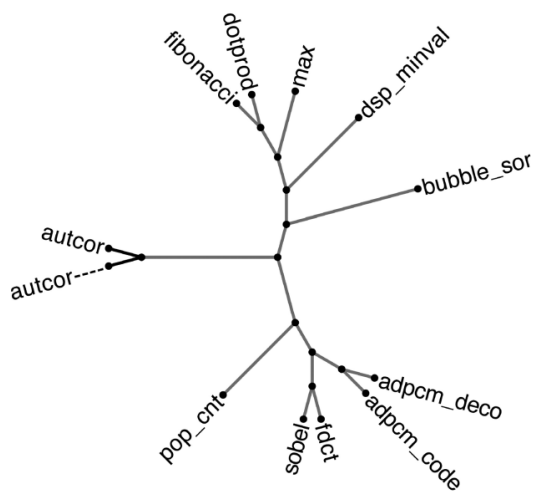

(b) DNA representation

Fig. 11. Phylogenetic trees generated from different input sources for the function autcor-

separately applied to the target function, adopting one that returns the best speedup. The approach based on the closest reference function (immediate neighbor) obtained the worst speedup $(1.18 \times)$. It indicates that the sequence of the closest functions does not always contain the necessary optimizations for a given target function. The employment of all the sequences of the cluster also did not achieve the mean speedup of DSE strategies, but reached values close to the ones achieved by Clean $(1.31 \times$ against $1.33 \times)$. However, this approach does not eliminate the need for clustering, since it uses the reference functions of the clusters generated through the selection method.

\subsection{Impact of the Input Representation on the DSE Performance}

In order to investigate the relevance of DNA in identifying the important code structures, other forms of representation of the source code were used as input to the selection method, and the results were compared with those obtained with the use of DNA. In this article, we performed clusters from source code and the Assembly code generated by ReflectC targeting the MicroBlaze processor.

Considering the geometric mean of the speedup achieved by DSE strategies based on the Clean algorithm and Clean-Insertion approach for each input representation and using the 46 functions, we observed that the influence of the input representation in the DSE performance is greater in Clean because of the algorithm simplicity. On the other hand, the speedups achieved by CI demonstrate the adaptability of this hybrid approach. In both approaches, the use of DNA and Assembly code achieved higher geometric mean speedups $(1.33 \times$ and $1.42 \times$, respectively), indicating that these input representations identify the similar structural patterns between codes. However, the DNAs are much smaller than their equivalents in Assembly (the file size with all DNA codes is $5.8 \mathrm{~KB}$, whereas the corresponding with Assembly codes is $86.6 \mathrm{~KB}$ ). Smaller files favor the compression process in the distance matrices computation. Furthermore, the use of DNA allows a clustering-based method independent of the compiler. The exploration based on source code achieved the worst values of speedup $(1.24 \times$ using Clean and $1.41 \times$ using CI).

Analyzing the generated phylogenetic trees, it was possible to observe that the source code input was not adequate to represent the similarity between codes in several situations, justifying why this representation is not suitable for pattern mining. An emblematic example can be seen in the phylogenetic trees of the autcor- shown in Figure 11. In the tree from the source code (Figure 11(a)), there are four internal nodes between the functions autcor_— and autcor, clustering them in different groups. 
Therefore, the selection method is not able to identify the similarity between these functions, even when their codes are almost identical. They only differ with respect to the length of the autocorrelation sequence used in the computation (10 in autcor and 16 in autcor- - ). The tree generated from the DNA (Figure 11(b)) adequately represents the similarity between these functions.

\subsection{Analysis of the DNA Encoding}

The DNA of the function is a simplified intermediate representation of its source code, which is encoding by a set of translation rules. The choice of these rules can influence the level of code details represented in the DNA code. Our approach uses the same rules used in Sanches et al. [2011]. These rules are used to represent only loops, discarding the remaining code. Nested loops are presented on the same line of the file, whereas adjacent loops are represented in separate lines.

Experiments were performed to investigate the influence of the DNA encoding only loops or entire code of the function in the performance achieved by the DSE. The DSE strategy based on the Clean algorithm was used, since it is more sensitive to changes than CI. Furthermore, the inclusion of new rules is also studied in order to verify the existence of any relevant information/structure that is not addressed in the default DNA. We tested new rules representing if structure; arithmetic operators, including operators of decrement and simplified (e.g., "-="); data types in detail (symbols for int, char, float, and double) or simplified form (symbol for integer and floating-point variables); data modifiers; function calls; constants; and pointer operators (“*” and "\&"). Each new rule was evaluated separately in the original set of rules. Finally, we also evaluate a complete set formed by combining all tested rules.

The DSE performance was very close in all evaluated configurations, with a small oscillation around 1.31 and 1.33. The DNAs (of only loops or full code) conduct to a similar behavior, with some specific variations, indicating that the effect of rule sets is stable in relation to how they are applied; that is, their effect is similar when compared to performance of the original rule set applied in the same way. The speedups achieved through encoding only loops were slightly better than those obtained from the DNA of the whole code (average difference about 0.006 and maximum value of 0.012 ), except for the full set (difference of -0.004). This validates the strategy currently employed to deal with functions whose intense processing is in the body of loops, as in image and signal processing domains.

The best DSE performance for DNA generated from the loops is achieved through the addition of the symbols for each data type (detailed representation) in the original set $(1.331 \times)$. The original set was also robust, obtaining a similar value $(1.326 \times)$. The adoption of symbols to represent the pointer operators and function calls results in the same speedup as the original DNA. The employment of other rules resulted in a small reduction of the mean speedup, and the worst performance was obtained from the set formed by all rules $(1.306 \times)$. In fact, when more information is added to the rule set, the DNA representation will be closer to the source code, which is not a good input to our clustering process. When the DNA generated represents the entire code, the original set of the translation rules achieves the best geometric mean speedup with DSE $(1.322 \times)$, whereas the representation of all constants shows the worst results $(1.307 \times)$. Based on the observed results, we conclude that the default set of rules is good enough for our purpose, especially considering only integer-type variables.

\subsection{Impact of Distance Metrics and Compressors in the Similarity Identification}

Experiments were performed in order to verify the influence of the scheme for calculating the dissimilarity matrices in our cluster-based approach. Here, we evaluate the use of the different compressors in the distance computation with the NCD metric, as 
well as the adoption of other distance metrics not based on compression to calculate the similarity from DNA codes.

The quality of the dissimilarity matrices computed through the NCD metric is related to the compressor adopted in the distance calculus [Cilibrasi and Vitanyi 2005]. Here, four different compression libraries were evaluated: bzlib (currently used), ppmdx, lzmax, and $z l i b$. These libraries are integrated into the CompLearn package [Cilibrasi et al. 2008], which is used by the selection method to calculate the matrix of distances. They were evaluated according to the performance achieved by Clean and its compression degree, measured from the compression of a file with the DNA code of all the functions (reference and target). The experimental results show that the compressors exhibit a similar behavior. The higher compression rates were achieved using the compressors lzmax and $z l i b$ ( $87.6 \%$ for both), whereas bzlib and ppmdx resulted in a rate of $86.9 \%$ and $85.3 \%$, respectively. In terms of performance, bzlib resulted in the best speedup $(1.33 \times)$ and ppmdx conducted to the worst value $(1.31 \times)$, whereas the other compressors (lzmax and $z l i b)$ resulted in a speedup of $1.32 \times$.

We also evaluated different distance metrics in the computation of dissimilarity matrices. Given that the DNA code is a sequence of symbols, we adopted distances able to deal directly with strings, which are provided in the package stringdist for the $\mathrm{R}$ language [van der Loo 2014]. They are categorized in string distances based on edits (Levenshtein - LV, Damerau-Levensthein - DL, optimal string alignment - OSA, and longest common substring - LCS) and substring granularity ( $q$-gram, cosine, Jaccard distance). In general, any other distance metric overcame NCD. With respect to Clean, distances as cosine $(1.32 \times)$ and $q$-gram $(1.31 \times)$ obtained values close to NCD $(1.33 \times)$. These two metrics are similar, since they deal with the occurrence of substrings in the DNA. The other distances resulted in speedups lower than $1.30 \times$. The speedups achieved by CI are very similar, around $1.42 \times$ for all distance metrics, except for the cosine that resulted in $1.41 \times$, showing again the adaptability of the hybrid approach.

\subsection{Analysis of the Reference Set}

The selection method employs a set of reference functions in the clustering of the new function. Therefore, the clustering efficiency is directly related to the representativity of this reference set. In order to reach a good coverage of the function space, the reference set should be formed by well-distributed points in the function space and each point must be significant within its region. Since an exhaustive search for the best set is unfeasible, criteria to support a systematic choice of the reference functions should be defined. In this article, reference set size was fixed to 11 functions to simplify the search space and facilitate the comparison of results. Besides, different reference sets were generated according to six different criteria of selection, as follows:

(1) Random sampling (RS): functions are randomly selected from the total 57 functions. In this strategy were evaluated 30 different sets.

(2) Best overall coverage (BOC): selecting functions, from all functions used in experiments, whose combination of their sequences covers the maximum number of distinct compilation passes. Here, 22 sets were generated, each containing 33 compilation passes.

(3) Best coverage of a random subset (BCR): same as previous, but considering only a subset of 26 functions randomly selected from the 57 functions. The strategy evaluated 30 sets with a different number of distinct passes.

(4) Best coverage per cluster (BCC): clustering all 57 functions into 11 groups using the Fast Newman algorithm [Newman 2010]. This algorithm is able to identify communities in complex networks and it was used to determine the clusters from the phylogenetic tree topology generated considering all functions. For each cluster, 


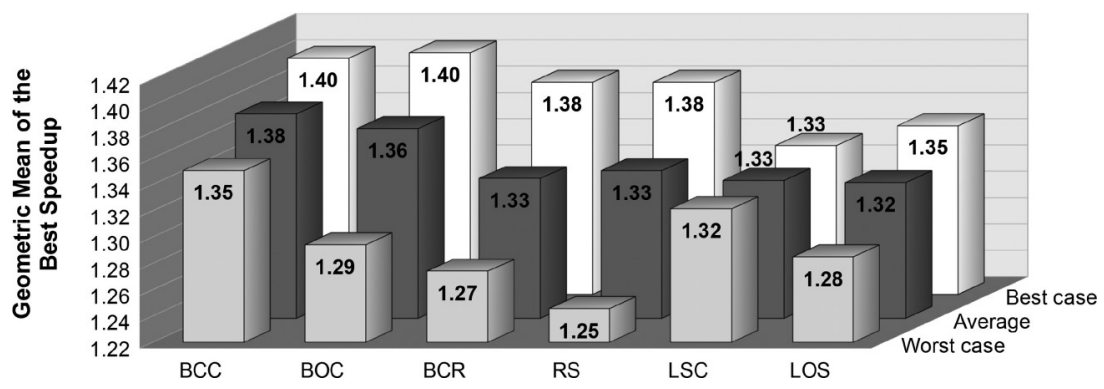

Fig. 12. Behavior of the selection criteria of the reference functions with respect to speedup achieved by the DSE based on the Clean algorithm.

a function is selected in order to maximize the number of distinct passes in the reference set. This criterion led to 128 sets with 29 passes each.

(5) Largest sequences per cluster (LSC): same as previous, but selecting a function with sequence with largest length from each cluster. The criterion generated eight sets.

(6) Largest overall sequences (LOS): selecting functions with sequences with the largest length considering all available functions. This strategy evaluated 32 sets.

A total of 258 different sets were evaluated in our experiments. The purpose is to research the impact of the selection criteria in the performance achieved by the DSE. Every set generated was individually applied to the exploration of target functions through the Clean algorithm. As different amounts of potential reference sets were generated in each selection strategy, the comparison between them was performed using the best and worst cases, average, and standard deviation. The geometric mean speedup achieved by Clean from each criterion used in the selection of the reference functions is highlighted in Figure 12.

The strategies based on randomness achieved in the worst cases $1.27 \times$ with the criterion based on the best coverage of a random subset and $1.25 \times$ with the random sampling, and they present a large variance between the values of their sets, which is evidenced by the standard deviation values ( 0.03 in both cases). The highest geometric mean speedup achieved with both these strategies was $1.38 \times$, which is lower than the best values obtained with the approaches based on the best coverage and higher than the best values resulting from the strategies that use the functions with the longest sequences. The worst cases for the two strategies based on long sequences were higher than the random strategies $(1.28 \times$ when considering all functions and $1.32 \times$ for clusterbased selection). These strategies also had a lower oscillation between performances of their sets (deviation standard of 0.02 and 0.004 , respectively), with similar means to those obtained by random approaches. However, they resulted in lower values among the best cases $(1.35 \times$ in overall criterion and $1.33 \times$ per cluster). The adoption of the best coverage criteria from the clusters and all functions (overall) achieved the highest mean speedups $(1.40 \times$ for both) and average $(1.38 \times$ in the coverage based on cluster and $1.36 \times$ in the overall coverage). However, the worst set of the overall coverage obtained a geometric mean speedup of $1.29 \times$, which is lower than those resulting from the approaches based on clusters $(1.35 \times$ in the best coverage per cluster and $1.32 \times$ in the largest sequences per cluster). In addition, there is a large variation and a standard deviation of 0.03 between the best and the worst speedups obtained from their sets. On the other hand, the strategy based on best coverage per cluster has a standard deviation of 0.01 , indicating a better balance between its sets. From the noted behavior, we conclude that the selection of a function of each cluster aiming to ensure 
the best coverage of the compiler passes is the most appropriate to systematically choosing a good reference set.

\subsection{Analysis of the Design Decisions}

In general, we conclude that the decisions and choices used to implement the selection process in our DSE environment were adequate. Using DNA as input representation is enough to effectively identify the important code properties. The DSE performance from the DNA clusters was similar to the clustering based on Assembly code without optimizations and more effective than one obtained from the source code. As the DNA code is smaller than its equivalent Assembly and it is independent of the compiler, it is more appropriate to be used in our approach. The translation rules currently employed in DNA encoding were the best among the sets of rules evaluated, returning the best speedups (or close to them). The experiments show that the DNA based on loop bodies is enough to represent the relevant elements of the source code. The differences of performance achieved between the application of the translation rules over the loop bodies or using the whole code were very subtle. However, even using different sets of DNA, the application of rules just inside the loops were consistently better than applying them over the entire code. Therefore, considering the set of functions evaluated, the strategy to use the DNA representation of only the body of the loops seems to be more adequate. The NCD [Cilibrasi and Vitanyi 2005] achieved the best geometric mean speedups for DSE strategies among the evaluated distance metrics. However, other string distance metrics based on symbols and substring existence also resulted in a good overall performance. On the other hand, edition-based metrics showed some difficulty in identifying good clusters, despite reaching values close to the ones obtained by the DSE scheme based on GA and exploring the full space for some individual functions. Besides, the compressor evaluated in the NCD computation does not affect significantly the performance of our DSE approach. Finally, we researched a systematic procedure to select a representative reference set from a pool of functions. In the best selection strategy, an initial clustering is performed in which all the functions of the pool are grouped in $k$ clusters, where $k$ is the specified size of the reference set. Subsequently, a function of each cluster is selected aiming to ensure the best coverage of the optimization passes. This selection procedure resulted in higher performance when compared to the other evaluated strategies, considering the worst, the average, and the best cases, as well as a small variation between the maximum and minimum values of the geometric mean speedup achieved by their evaluated sets.

\subsection{Expanding Our Evaluation to Other Benchmarks}

In addition to functions from the DSP and image processing benchmark repositories from Texas Instruments, we also evaluated our approach using quick sort, Dijkstra, blowfish coder, and blowfish decoder functions from the MiBench benchmark suite [Guthaus, et al. 2001]; a hot-spot function from an industrial application (filter Subband, part of an MPEG audio encoder), which was provided in the context of the REFLECT project [Cardoso et al. 2013]; and compress, histogram, edge detection, and $l p c$ applications from the UTDSP benchmark suite [Lee 2002]. The experiments with Mibench's functions and the filter Subband were performed in order to evaluate the effectiveness of our approach to optimize codes from other computing domains. The adoption of UTDSP applications aims to evaluate the behavior of our approach when applied to more complex functions. In this context, the key functions of each application were compiled separately with different specific optimization sequences, and their optimized codes were combined.

Figure 13 outlines the best speedups achieved with DSE using GA, Clean, and Clean-Insertion algorithms. The last bar in the chart of Figure 13 shows the respective 


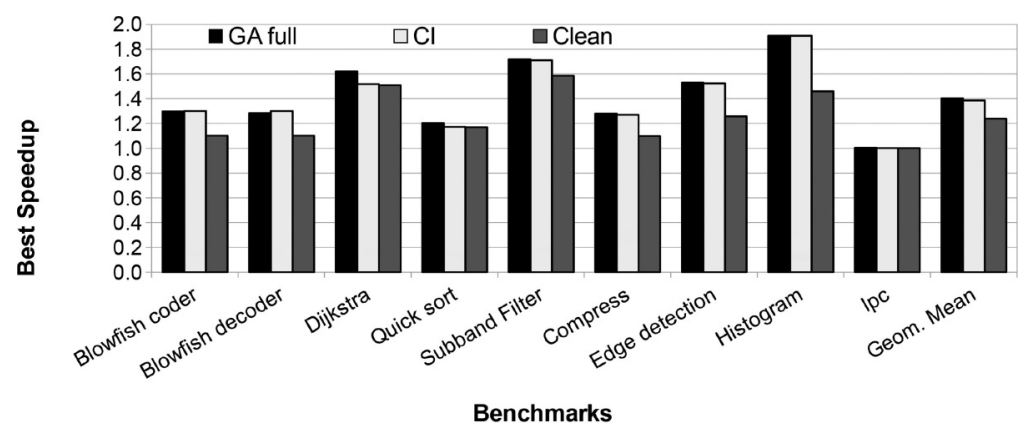

Fig. 13. Speedups achieved for each new benchmark using nondominated DSE strategies.

geometric mean. In general, it was possible to observe an improvement in the performance of the functions/applications after optimization for all the researched strategies. The unique exception was the $l p c$ application, which did not achieve any speedup no matter the strategy used. GA using the full-space strategy achieved the best results in all cases. CI resulted in very close speedups to GA. Between the three strategies, Clean resulted in the worst performance. The geometric mean speedups achieved for GA Full, CI, and Clean were $1.43 \times, 1.41 \times$, and $1.28 \times$, respectively. This behavior was compatible with that observed in the benchmarks initially evaluated. Due to the complexity increment in the new evaluated functions/applications compared with the initial benchmark, the execution time was much higher than in the previous functions. However, compatible with what was observed in the early analysis, Clean was the fastest strategy and GA with full space was the slowest. The average of the execution time achieved for GA Full, CI, and Clean were 27,778, 1,357, and 62 seconds, respectively. Therefore, we can conclude that the general panorama was maintained even using more complex applications or functions from other domains: (1) GA achieves the best speedups, but CI returns almost the same performance with a much faster exploration, and (2) Clean returns the worst speedups, but it is extremely fast.

\subsection{Extending the Comparative Analysis}

The adoption of different compiler environments and target architectures makes it difficult to compare the results between published works. In order to overcome this problem, we performed experiments using a compilation environment based on the LLVM compiler infrastructure (version 3.5) [Lattner and Adve 2004] and targeting a LEON3 processor. Both LLVM and LEON3 are open-source projects and widely used. Therefore, this allows one to reproduce the compiler environment and comparisons with other work efforts can be made. Besides, the performance can be compared with -O compilation strategies. Here, we compare the speedups achieved using our DSE strategies with those obtained with LLVM -O2 and with the best speedups achieved using the top 10 sequences presented in Purini and Jain [2013].

Figure 14(a) presents the speedups achieved with optimization sequences found using Clean and CI strategies in relation to LLVM -O2. Comparing CI and -O2 speedups, $74 \%$ of all 54 compiled codes achieve better speedups with CI, -O2 overcame CI in 17\% of them, and the other $9 \%$ resulted in the same performance with both optimization approaches. The geometric mean of the speedup in relation to -O2 achieved with CI considering all 54 codes is $1.07 \times$. If we observe only the 10,20 , and 30 best results, the geometric mean speedup achieved with CI (black lines) is $1.49 \times, 1.32 \times$, and $1.24 \times$. Considering the speedups achieved using Clean and -O2, 54\% of the codes achieve better speedups with Clean, -O2 overcame Clean in $37 \%$ of them, and $9 \%$ of codes resulted 


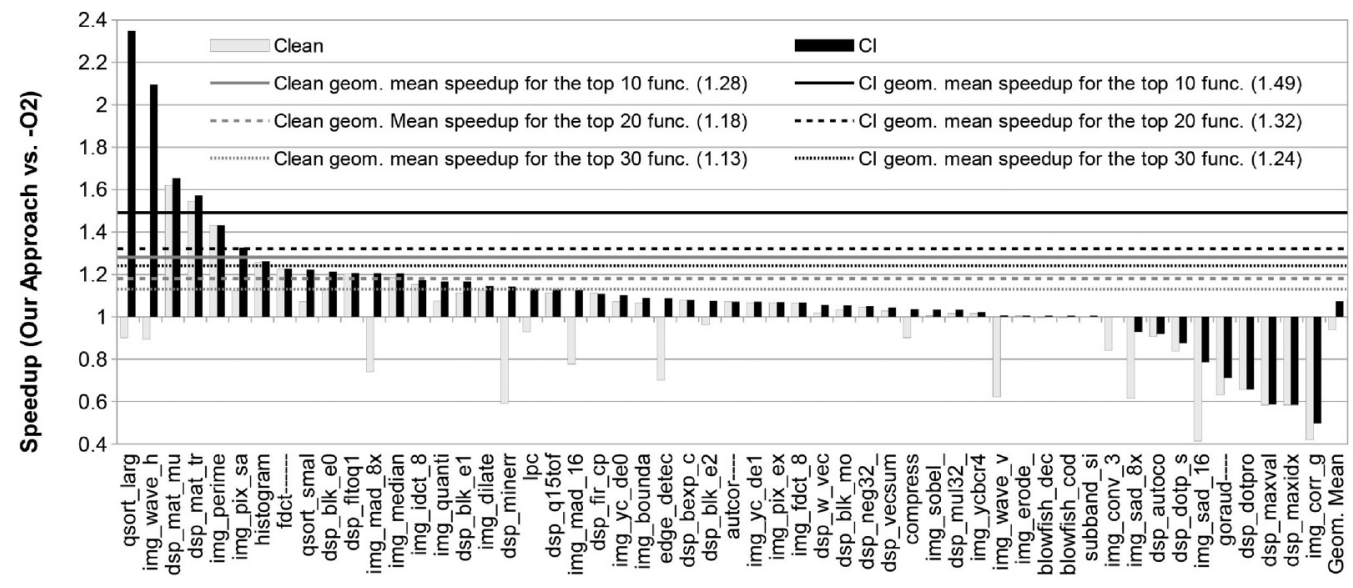

(a) Our approach versus -O2

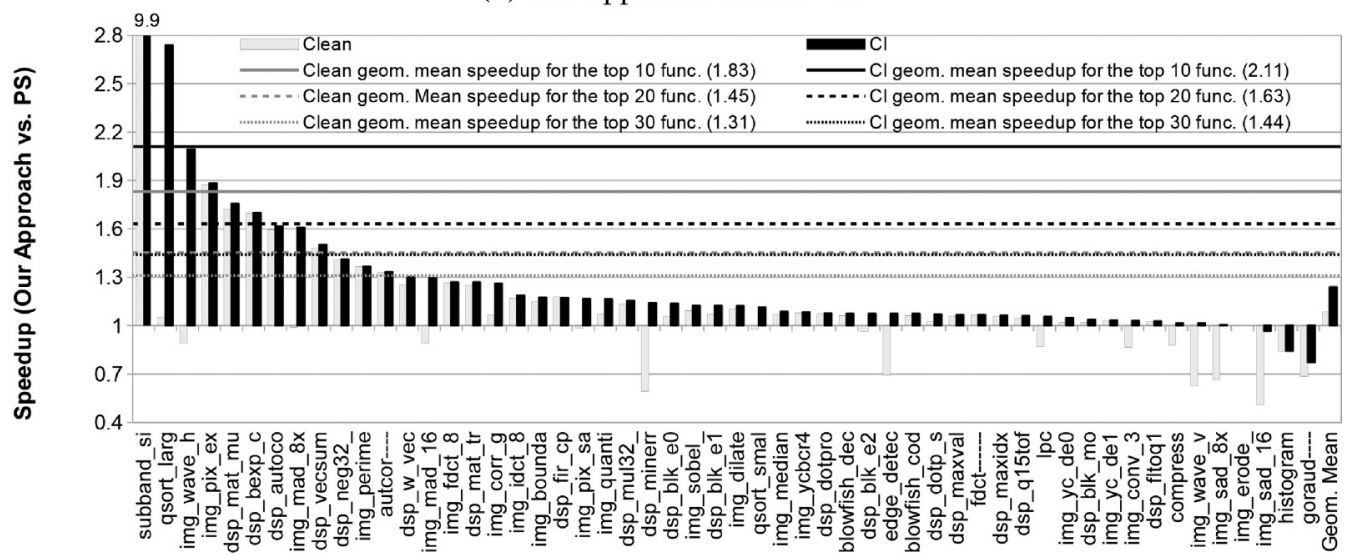

(b) Our approach versus the best 10 sequences proposed in Purini and Jain [2013]

Fig. 14. Speedup achieved from LLVM compiler infrastructure using Clean and CI.

in the same performance with both approaches. The geometric mean of the speedup in relation to -O2 is 0.94 . However, when we consider only the 10,20 , and 30 best results, the geometric mean speedup achieved with Clean (gray lines) is $1.28 \times, 1.18 \times$, and $1.13 \times$. Therefore, we can conclude that in general, CI achieves higher speedups than the -O2 option using the LLVM compiler, corroborating the good performance found previously with ReflectC. However, Clean presented a worse performance, and its results are similar to the -O2 option. We believe this decay of performance from ReflectC to LLVM experiments can be justified by the fact that LLVM employs more optimization passes (124 vs. 49), and some of them, such as loop unrolling, have a strong semantic dependence. On the other hand, our clustering process is not considering semantic information and it is fully syntactically guided.

Figure 14(b) shows the speedup achieved using DSE strategies in relation to the exhaustive search using the 10 best sequences presented in Purini and Jain [2013]. Considering the CI performance and the best speedup achieved from Purini's sequences (PS), $91 \%$ of the compiled codes achieve better speedups with CI, PS overcame CI in $6 \%$ of them, and the other $3 \%$ of codes resulted in the same speedup with both. The geometric mean of the speedup achieved with CI in relation to PS is $1.24 \times$. If we 
consider only the 10,20 , and 30 best results, the geometric mean speedup achieved with CI is $2.11 \times, 1.63 \times$, and $1.44 \times$. Comparing Clean and PS, $69 \%$ of the codes achieved better speedups with Clean, while PS returned better speedups in 30\%. The geometric mean speedup in relation to PS considering all 54 codes is $1.09 \times$. Considering only the 10,20, and 30 best speedups, the geometric mean speedup achieved with Clean is $1.83 \times, 1.45 \times$, and $1.31 \times$. In general, we can conclude that using the LLVM compiler, CI achieved much better results than PS: more than $90 \%$ of the codes were improved in relation to PS. Clean also presented a good performance and its results overcame PS in almost $70 \%$ of the evaluated codes.

\section{RELATED WORK}

It is a common practice to apply the same set of optimizations in a fixed order, previously hand-tuned by compiler writers based on their experience and insight [Kulkarni and Cavazos 2012]. However, different code fragments may require a specific ordering of optimizations to obtain the best performance for a given platform. Compilers for embedded computing systems are more dependent on code transformations and optimizations as the target architectures used have typically more constraints. For this kind of system, nontrivial compiler sequences are usually needed to achieve the required results. Given the large number of compiler optimizations, the design space is vast. The research of techniques to efficiently and effectively search this space is a hot topic of research.

The identification of compiler sequences generally relies on iterative compilation, predictive-based methods, or both. Machine-learning techniques have been commonly employed to improve the search for the best optimization sequences for specific applications. A genetic-algorithm-based approach is used in Cooper et al. [1999] to find optimization sequences in order to reduce the object code size. Randomized search algorithms have been used to identify suitable compilation sequences [Cooper et al. 2006]. A system called COLE is presented in Hoste and Eeckhout [2008]. It uses a multiobjective evolutionary algorithm based on SPEA2 [Zitzler et al. 2001] to automatically find Pareto optimal settings of optimization flags for the entire application in the gcc compiler. The work in Chen et al. [2012] experimentally demonstrated that there exists at least one program-level compiler optimization selection that achieves at least $83 \%$ or more of the best-known speedup across 1,000 different datasets for each of the 32 programs considered. The authors suggest that optimizing programs across datasets might be much easier than previously anticipated. Recent research has proposed heuristic techniques based on code characterization to quickly customize sequences for individual functions during static and dynamic compilation. In Cavazos and O'Boyle [2006], a predictive model based on logistic regression uses the features extracted from the function to select the optimization to apply in the Jikes RVM Java compiler, using a fixed-order of compiler passes. This work was extended in Kulkarni and Cavazos [2012], applying method-specific optimization orderings. The authors address the phase-ordering problem as a Markov process, where the characterization of the current state of the code is used as input of the neural network for selecting optimizations to apply during dynamic compilation. Support vector machines are employed in Sanchez et al. [2011] to derive function-specific compilation strategies for the IBM J9 Java compiler. These approaches reveal that the use of analytical models and cost functions based on certain code features may allow good predictors and may truly contribute to the identification of compiler sequences. A Bayesian-network-based model was used in Ashouri et al. [2014] to provide a probability distribution for the compilation passes from the machine-independent features dynamically extracted from the application. These probabilities guide the iterative search to autotuning of the optimization flags of the gcc compiler aiming at the speedup of the application execution. 
All previous approaches are focused on the DSE. Both static and dynamic heuristic approaches aid the search for the best compiler optimizations. On the other hand, our approach focuses on the selection of potential compiler passes in order to restrict/reduce the search space for DSE. Therefore, our work is orthogonal to these approaches as search space pruning can be seen as a separate task of the exploration process. Generally, it can be used as an important initial step of DSE. Moreover, it is independent of features extracted from the source code, although they can be used as input data during the clustering phase to improve the algorithm's accuracy.

Several techniques have been used to reduce the space of potential candidate solutions. A statistical analysis of the effect of compiler options is used in Haneda et al. [2005] to prune the search space and find a single compiler optimization sequence for a set of programs that perform better than the standard settings used in gcc. Agakov et al. [2006] proposed a biased search technique using Markov models to limit the search space, increasing the speed of iterative optimizations. This methodology is able to indicate, for a given program, the areas of the solution space where the search should be focused. Moreover, it is independent of the solution space, the search algorithm, and the compiler/optimizer used. The work in Kulkarni et al. [2007] uses several machinelearning algorithms with aggressive pruning through exhaustive enumeration of all function instances for a set of programs, observing that search techniques such as GAs achieve gains very close to best performance. This work was extended in Kulkarni et al. [2009] and Kulkarni et al. [2010], where they compare the ability of GA-based and function-level searches to find the best optimization sequences for their VPO compiler. Jantz and Kulkarni [2013] employ an exhaustive DSE algorithm presented in previous work, adding independence characteristics of optimization passes in the pruning technique and performing searches in multiple stages over smaller disjoint subsets of optimizations. PEAK, an automated performance tuning system, is presented in Pan and Eigenmann [2008]. It uses three heuristic algorithms to select good compiler optimization settings. It performs an initial space pruning through the withdrawal of transformations with negative performance effects to speed up the search. Unlike our approach, previous works are based on information about compiler pass iterations in preliminary research. Down-sampling techniques are used in Purini and Jain [2013] to reduce the search space to a few good optimization sequences (around 10) with respect to representative instances of program classes. These sequences are applied in the new program and the best one is selected. This work differs from our approach, which aims at searching a customized sequence for a specific function. In Fursin et al. [2011], a gcc-based framework (Milepost GCC) is used to automatically extract program features and learn the best optimizations across programs and architectures. Their framework uses a probabilistic prediction model to correlate the new program with the closest one seen earlier to apply a customized optimization combination. Although this work is similar to our approach, it focuses on the phase selection problem where the application order of optimizations is fixed, while our method addresses the phase-ordering problem. Moreover, our approach does not need any training and is based on clustering and simple DSE schemes. The idea is that the code patterns extracted by clustering can aid in the appropriate selection of passes, which can be used in DSE strategies to conduct/suggest efficient compiler sequences.

\section{CONCLUSIONS}

The selection of the sequence of compiler optimizations can have a significant impact on performance, and it is architecture and application dependent. Therefore, the adoption of an efficient Design Space Exploration (DSE) scheme to aid embedded system developers is of paramount importance. This article presented a DSE approach that uses a clustering-based selection method for reducing the number of compiler optimizations 
used during the exploration of optimization sequences. We analyzed DSE algorithm alternatives to employ in our clustering-based approach. Experiments showed that the use of our approach results in a significant reduction of the search space and makes feasible the use of simple DSE algorithms. Approaches based on the Clean algorithm resulted in a good tradeoff between program optimization achievements (performance improvements) and DSE execution time. Particularly, the performance improvements achieved are comparable to close-to-optimal compilation sequences found by a Genetic Algorithm (GA) using the full optimizations set. When compared to the results achieved by a DSE strategy using a GA and targeting a Xilinx MicroBlaze processor, our approach using a simple and fast algorithm (Clean algorithm) allowed significant reductions of DSE execution time (around 286 $\times$ ) at the same time that it achieved performance improvements only $10 \%$ below the ones provided by GA, guaranteeing performance improvements of $33 \%$ over the baseline for the 46 functions from the TI benchmarks used in the experiments. The Clean-Insertion algorithm resulted in performance improvements similar to those found by the GA approach (42\% and $43 \%$, respectively), with an $18 \times$ faster DSE execution time (364 seconds compared to 108 minutes of GA).

The additional experiments carried out using the LLVM compiler and targeting a LEON3 processor have also shown the importance of our approach when other compilation environments are used. Comparing its performance with other optimization approaches (e.g., -O2), it was possible to observe that our approach overcame LLVM -O2 using the Clean-Insertion algorithm and achieved similar performance to -O2 when using the Clean algorithm. Specifically, our approach achieved geometric mean speedups of $1.49 \times, 1.32 \times$, and $1.24 \times$ when considering the best 10,20 , and 30 functions, respectively. When considering the entire benchmark set consisting of 54 functions/applications, our approach achieved a more modest average performance improvement (7\%).

Ongoing work is focusing on probabilistic heuristics that explore each clusteringbased set of optimizations and on exploring sequences of compiler optimizations in the context of hardware generation targeting FPGAs. For future work, we plan to compare our DSE approach to other machine-learning approaches to see if our DSE approach provides a benefit over the state of the art. In the analysis of the distance metrics, it was observed that even metrics that resulted in executables with a lower mean performance also achieved speedups for some functions that are very close or equal to those obtained when exploring the full space with a genetic algorithm. Thus, the combination of phylogenetic trees generated from a preselected group of distances, like ensemble or consensus trees, may be a promising research avenue. As the current DNA representation is fully based on syntax-oriented translation rules, we also intend to research the impact of using other DNA translation rules (semantically based and possibly including also data dependence information).

\section{REFERENCES}

ACE. 2012. CoSy Compiler Development System. Retrieved from http://www.ace.nl/compiler/cosy.html.

Aeroflex. 2003. TSIM2 ERC32/LEON Simulator. Retrieved from http://www.gaisler.com/index.php/products/ simulators/tsim.

Aeroflex. 2005. LEON3 Processor. Retrieved from http:/www.gaisler.com/index.php/products/processors/ leon3.

F. Agakov, E. Bonilla, J. Cavazos, B. Franke, G. Fursin, M. F. P. O’Boyle, J. Thomson, M. Toussaint, and C. K. I. Williams. 2006. Using machine learning to focus iterative optimization. In Proc. of the Int. Symp. on Code Generation and Optimization (CGO'06). 295-305.

L. Almagor, K. D. Cooper, A. Grosul, T. J. Harvey, S. W. Reeves, D. Subramanian, L. Torczon, and T. Waterman. 2004. Finding effective compilation sequences. In Proc. of the ACM Conf. on Languages, Compilers, and Tools for Embedded Systems (LCTES'04), Vol. 39. 231-239. 
A. H. Ashouri, G. Mariani, G. Palermo, and C. Silvano. 2014. A Bayesian network approach for compiler autotuning for embedded processors. In IEEE 12th Symp. on Embedded Systems for Real-time Multimedia (ESTIMedia'14). 90-97.

J. M. P. Cardoso, P. C. Diniz, J. G. F. Coutinho, and Z. M. Petrov (Eds.). 2013. Compilation and Synthesis for Embedded Reconfigurable Systems: An Aspect-Oriented Approach. Springer.

J. M. P. Cardoso, T. Carvalho, J. G. F. Coutinho, W. Luk, R. Nobre, P. Diniz, and Z. M. Petrov. 2012. LARA: An aspect-oriented programming language for embedded systems. In Proc. of the 11th ACM Int. Conf. on Aspect-Oriented Software Development (AOSD'12). 179-190.

J. Cavazos and M. F. P. O'Boyle. 2006. Method-specific dynamic compilation using logistic regression. In Proc. of the ACM Int. Conf. on Object-Oriented Programming Systems, Languages, and Applications (OOPSLA'06). 229-240.

Y. Chen, S. Fang, Y. Huang, L. Eeckhout, G. Fursin, O. Temam, and C. Wu. 2012. Deconstructing iterative optimization. ACM Trans. Archit. Code Optim. 9, 3 (2012), 21:1-21:30.

A. R. Cilibrasi and A. P. Vitanyi. 2005. Clustering by compression. In Proc. of the IEEE Trans. Information Theory (AOSD'12), Vol. 51. 1523-1545.

A. R. Cilibrasi, A. L. Cruz, S. de Rooij, and M. Keijzer. 2008. CompLearn Toolkit. Retrieved from http://www.complearn.org/.

K. D. Cooper, P. J. Schielke, and D. Subramanian. 1999. Optimizing for reduced code space using genetic algorithms. In Proc. of the ACM Workshop on Languages, Compilers, and Tools for Embedded Systems (LCTES'99). 1-9.

K. D. Cooper, A. Grosul, T. J. Harvey, S. Reeves, D. Subramanian, L. Torczon, and T. Waterman. 2006. Exploring the structure of the space of compilation sequences using randomized search algorithms. $J$. Supercomput. 36, 2 (2006), 135-151.

J. Felsenstein. 2003. Inferring Phylogenies. Sinauer Associates.

G. Fursin, Y. Kashnikov, A. Memon, Z. Chamski, O. Temam, M. Namolaru, E. Yom-Tov, B. Mendelson, A. Zaks, E. Courtois, F. Bodin, P. Barnard, E. Ashton, E. Bonilla, J. Thomson, C. Williams, and M. O’Boyle. 2011. Milepost GCC: Machine learning enabled self-tuning compiler. Int. J. Parallel Program. 39, 3 (2011), 296-327.

D. E. Goldberg. 1989. Genetic Algorithms in Search, Optimization and Machine Learning. Addison-Wesley Longman.

M. R. Guthaus, J. S. Ringenberg, D. Ernst, T. M. Austin, T. Mudge, and R. B. Brown. 2001. MiBench: A free, commercially representative embedded benchmark suite. In Proc. of the IEEE Int. Workshop of the Workload Characterization (WWC'01). 3-14.

M. Haneda, P. M. W. Knijnenburg, and H. A. G. Wijshoff. 2005. Optimizing general purpose compiler optimization. In Proc. of the 2nd Conf. on Computing Frontiers (CF'05). 180-188.

K. Hoste and L. Eeckhout. 2008. Cole: Compiler optimization level exploration. In Proc. of the 6th Int. Symp. on Code Generation and Optimization (CGO'08). 165-174.

Q. Huang, R. Lian, A. Canis, J. Choi, R. Xi, S. Brown, and J. Anderson. 2013. The effect of compiler optimizations on high-level synthesis for FPGAs. In Proc. of the IEEE Int. Symp. on Field-Programmable Custom Computing Machines (FCCM'13). 89-96.

M. R. Jantz and P. A. Kulkarni. 2013. Exploiting phase inter-dependencies for faster iterative compiler optimization phase order searches. In Proc. of the Conf. on Compilers, Archit. and Synthesis for Embedded Systems (CASES'13). 7:1-7:10.

P. A. Kulkarni, M. R. Jantz, and D. B. Whalley. 2010. Improving both the performance benefits and speed of optimization phase sequence searches. In Proc. of the ACM Conf. on Lang., Comp., and Tools for Emb. Syst. (LCTES'10). 95-104.

P. A. Kulkarni, D. B. Whalley, and G. S. Tyson. 2007. Evaluating heuristic optimization phase order search algorithms. In Proc. of the IEEE Int. Symp. on Code Generation and Optimization (CGO'07). 157-169.

S. Kulkarni and J. Cavazos. 2012. Mitigating the compiler optimization phase-ordering problem using machine learning. In Proc. of the ACM Conf. on Object Oriented Programming Systems Lang. and Applications (OOPSLA'12). 147-162.

P. A. Kulkarni, D. B. Whalley, G. S. Tyson, and J. W. Davidson. 2009. Practical exhaustive optimization phase order exploration and evaluation. ACM Trans. Architect. Code Optim. 6, 1 (April 2009), 1:1-1:36.

C. Lattner and V. Adve. 2004. LLVM: A compilation framework for lifelong program analysis \& transformation. In Proc. of the Int. Symp. on Code Generation and Optimization (CGO'04). 75.

C. G. Lee. 2002. UTDSP benchmark suite. (2002). http://www.eecg.toronto.edu/ corinna/DSP/infrastructure/ UTDSP.tar.gz. 
M. Li and P. M. B. Vitanyi. 1997. An Introduction to Kolmogorov Complexity and Its Applications (2nd ed.). Springer-Verlag.

L. G. A. Martins, R. Nobre, A. C. B. Delbem, E. Marques, and J. M. P. Cardoso. 2014a. A clustering-based approach for exploring sequences of compiler optimizations. In Proc. of the IEEE Congress on Evolutionary Computation (CEC'14). 1-8.

L. G. A. Martins, R. Nobre, A. C. B. Delbem, E. Marques, and J. M. P. Cardoso. 2014b. Exploration of compiler optimization sequences using clustering-based selection. In Proc. of the ACM Conf. on Languages, Compilers, and Tools for Embedded Systems (LCTES'14). 63-72.

M. Newman. 2010. Networks: An Introduction. Oxford University Press.

Z. Pan and R. Eigenmann. 2008. PEAK: A fast and effective performance tuning system via compiler optimization orchestration. ACM Trans. Program. Lang. Syst. 30, 3 (2008), 1-17.

S. Purini and L. Jain. 2013. Finding good optimization sequences covering program space. ACM Trans. Architect. Code Optim. 9, 4 (January 2013), 56:1-56:23.

C. K. Roy, J. R. Cordy, and R. Koschke. 2009. Comparison and evaluation of code clone detection techniques and tools: A qualitative approach. Sci. Comput. Program. 74, 7 (May 2009), 470-495.

A. Sanches and J. M. P. Cardoso. 2010. On identifying patterns in code repositories to assist the generation of hardware templates. In Proc. of the 20th Int. Conf. on Field Programmable Logic and Applications (FPL'10). 267-270.

A. Sanches, J. M. P. Cardoso, and A. C. B. Delbem. 2011. Identifying merge-beneficial software kernels for hardware implementation. In Proc. of the Int. conf. on Reconfigurable Computing and FPGAs (ReConFig'11). 74-79.

R. Sanchez, J. Amaral, D. Szafron, M. Pirvu, and M. Stoodley. 2011. Using machines to learn method-specific compilation strategies. In Proc. of the Int. Symp. on Code Generation and Optimization (CGO'11). 257266.

Texas Instruments. 2003a. TMS320C64x DSP Library: Programmer's Reference. (2003).

Texas Instruments. 2003b. TMS320C64x Image/Video Processing Library. (2003).

M. P. J. van der Loo. 2014. The stringdist package for approximate string matching. The R Journal 6 (2014), 111-122.

T. Wheeler and J. Kececioglu. 2007. Multiple alignment by aligning alignments. In Proc. of the 15th ISCB Conf. on Intelligent Systems for Molecular Biology, Bioinformatics, Vol. 23. i559-i568.

E. Zitzler, M. Laumanns, and L. Thiele. 2001. SPEA2: Improving the Strength Pareto Evolutionary Algorithm. Computer Engineering and Networks Lab Technical Report TR-200. Swiss Federal Institute of Technology, Cambridge, MA.

Received May 2015; revised December 2015; accepted January 2016 